\title{
Neural circuit basis of aversive odour processing in Drosophila from sensory input to descending output.
}

\author{
Paavo Huoviala, ${ }^{1,2}$, Michael-John Dolan, ${ }^{1,3, \dagger}$, Fiona M. Love, ${ }^{2, \dagger}$, Philip Myers, ${ }^{1}$, Shahar Frechter, ${ }^{1}$, \\ Shigehiro Namiki, ${ }^{3}$, Lovisa Pettersson, ${ }^{4}$, Ruairí J.V. Roberts, ${ }^{2}$, Robert Turnbull, ${ }^{2}$, Zane Mitrevica, ${ }^{2}$, \\ Patrick Breads, ${ }^{3}$, Philipp Schlegel, ${ }^{2}$, Alexander Shakeel Bates, ${ }^{1}$, Tiago Rodrigues, ${ }^{1}$, Yoshinori Aso, ${ }^{3}$, Davi \\ Bock, ${ }^{3}$, Gerald M. Rubin, ${ }^{3}$, Marcus Stensmyr, ${ }^{4}$, Gwyneth Card, ${ }^{3}$, Marta Costa, ${ }^{2}$, Gregory S.X.E. \\ Jefferis, ${ }^{1,2, *}$ \\ ${ }^{1}$ MRC Laboratory of Molecular Biology, Cambridge, CB2 0QH, UK \\ 2 Drosophila Connectomics Group, Department of Zoology, University of Cambridge, Downing Street, Cambridge, CB2 3EJ UK \\ 3 Janelia Research Campus, Howard Hughes Medical Institute, USA \\ ${ }^{4}$ Department of Biology, Lund University, 22100 Lund, Sweden \\ $\dagger$ These authors contributed equally to this work \\ * Corresponding author: jefferis@mrc-lmb.cam.ac.uk
}

\begin{abstract}
Evolution has shaped nervous systems to produce stereotyped behavioural responses to ethologically relevant stimuli. For example when laying eggs, female Drosophila avoid geosmin, an odorant produced by toxic moulds. Here we identify second, third, and fourth order neurons required for this innate olfactory aversion. Connectomics data place these neurons in a complete synaptic circuit from sensory input to descending output. We find multiple levels of valence-specific convergence, including a novel form of axo-axonic input onto second order neurons conveying another danger signal, the pheromone of parasitoid wasps. However, we also observe extensive divergence: second order geosmin neurons connect with a diverse array of 80 third order cell types. We find a pattern of convergence of aversive odour channels at this level. Crossing one more synaptic layer, we identified descending neurons critical for egg-laying aversion. Our data suggest a transition from a labelled line organisation in the periphery to a highly distributed central brain representation that is then coupled to distinct descending pathways.
\end{abstract}

Microbes and parasites are a major driving force of natural selection in animals. As immunological defence is costly and reactive, it is better to avoid sources of infection whenever possible (1). Drosophila avoid laying eggs on food contaminated with harmful moulds. This avoidance is triggered by a volatile molecule, geosmin, sensed through a single olfactory receptor (Or56a) expressed in only $\sim 25$ sensory neurons (ORNs) per antenna $(2,3)$. The olfactory system, in both vertebrates and invertebrates, is a particularly shallow sensory modality where the sensory periphery is only two synapses away from higher brain areas important for organizing behaviour and forming memories (4). The clear behavioural significance of geosmin, together with Or56a ORNs being its sole dedicated sensor, make this 'labelled line' pathway an attractive target for studies of how sensory signals are transformed into innate, ethologically appropriate behavioural responses. Since the challenge of differentiating suitable from contaminated food substrates is ubiquitous and encompasses many issues in sensory processing, general principles are likely to emerge from these studies.
Building on the identification of Or56a sensory neurons (2), we traced the geosmin pathway deeper into the brain. Wild-type flies avoid geosmin in an egg-laying two-choice assay, and this avoidance is solely due to olfaction via the Or56a ORNs (2) (Figure S1, A and B). However, geosmin did not decrease egg-laying quantity in a no-choice situation (Figure S1C), suggesting that the phenotype arises from positional aversion. Or56a ORNs synapse onto uniglomerular DA2 projection neurons (PNs) in the antennal lobe. We identified a sparse driver line (R85E04-GAL4, Figure 1A (5)) that potentially labelled DA2 PNs. Driving the Halo-tag reporter (6) with Or56a-GAL4 and R85E04 verified that both sets of neurons target the same glomerulus (Figure S1D) while immunostaining confirmed that the PNs are cholinergic and excitatory (Figure S1F). In vivo electrophysiology revealed strong and highly selective responses to geosmin (Figure S1E). 'Labelled-line' encoding of geosmin is therefore retained at the second order PN level.

To demonstrate the functional role of these DA2 PNs, we silenced their synaptic output by expressing tetanus toxin (7) via R85E04. This completely abolished avoidance behaviour (Figure 1B) confirming that DA2 PNs are necessary for geosmin sensing. In summary, R85E04 labels the excitatory DA2 PNs postsynaptic to Or56a ORNs; these PNs respond strongly and selectively to geosmin, and are necessary for geosmin avoidance behaviour during egg-laying, a task of key ethological importance to the animal.

DA2 PNs project to two higher brain areas: the mushroom body (MB) and the lateral horn (LH). The former is thought to be involved in associative learning and the latter in innate behaviour. As expected, the MB appeared not to play a role in geosmin aversion (Figure 1B), so we focused on the LH. The axonal morphology of DA2 PNs shows notable similarities with several other aversive odour processing PNs (Figure 1C), particularly in the ventral-posterior LH (dashed line in Figure 1C), an area recently suggested to be important for egg-laying aversion (8). The similarity is especially striking with DL4 PNs, which are postsynaptic to Or49a/Or85f ORNs tuned to the sex pheromone of the parasitic wasp $L$. boulardi (9). This suggests the possibility of valence-based integration 
bioRxiv preprint doi: https://doi.org/10.1101/394403; this version posted November 4, 2020. The copyright holder for this preprint (which was not certified by peer review) is the author/funder, who has granted bioRxiv a license to display the preprint in perpetuity. It is made available under aCC-BY-NC 4.0 International license.

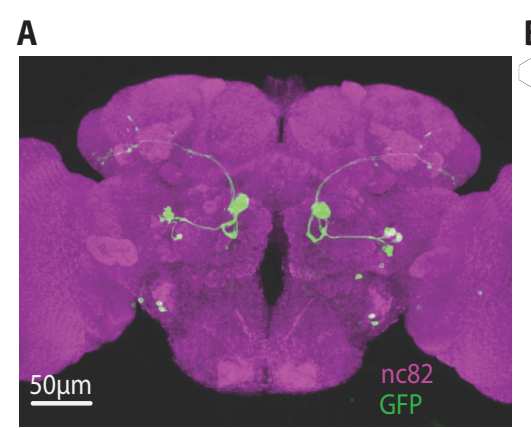

R85E04-GAL4

D

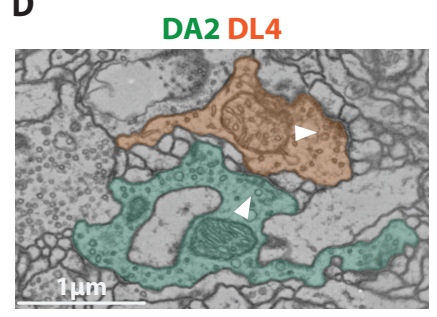

G1

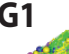<smiles>[Al]=C=[V]</smiles>
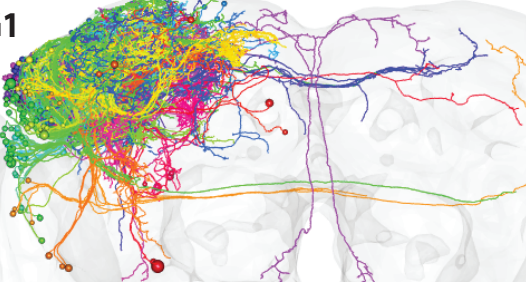

LHON

$\mathrm{n}=171$

$70 \%$ of DA2 synapses

H

LHONs: 55 clusters
B

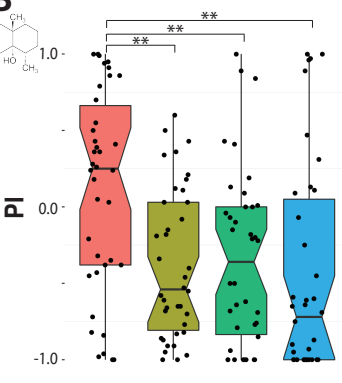

E
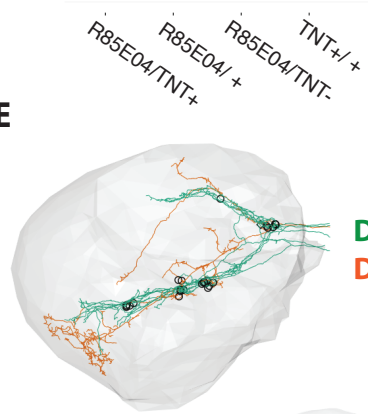

DA2

DL4

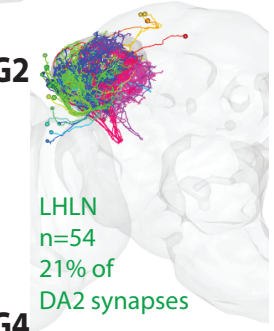

G4

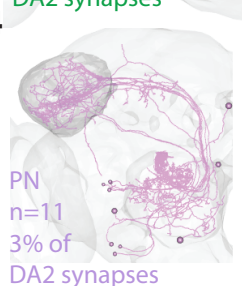

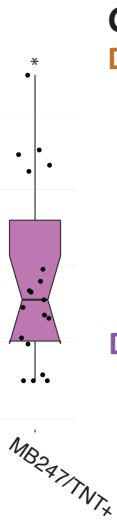

DL4 - iridomyrmecin

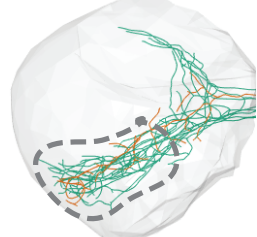

DL5 - benzaldehyde
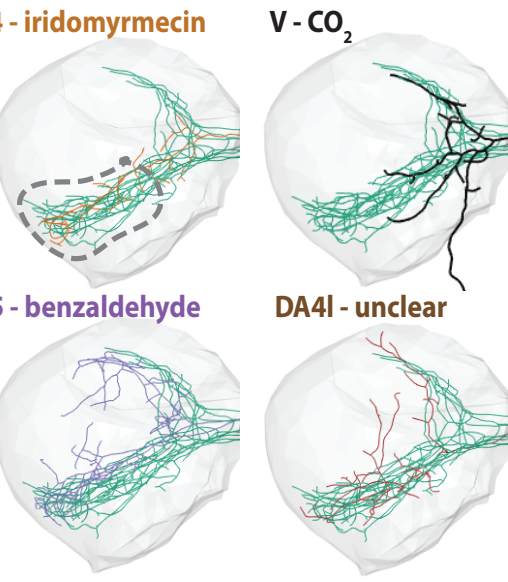

DA4I - unclear

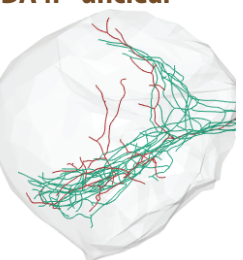

F $\mathrm{DC} 1$

DM5

VA6

DL3

DA3
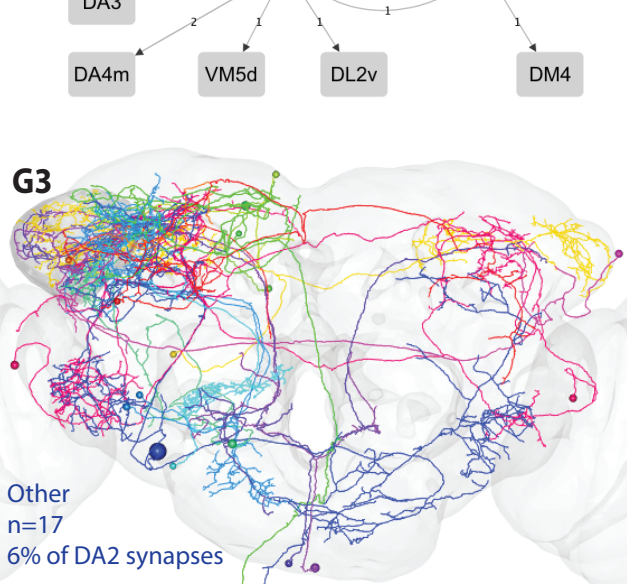

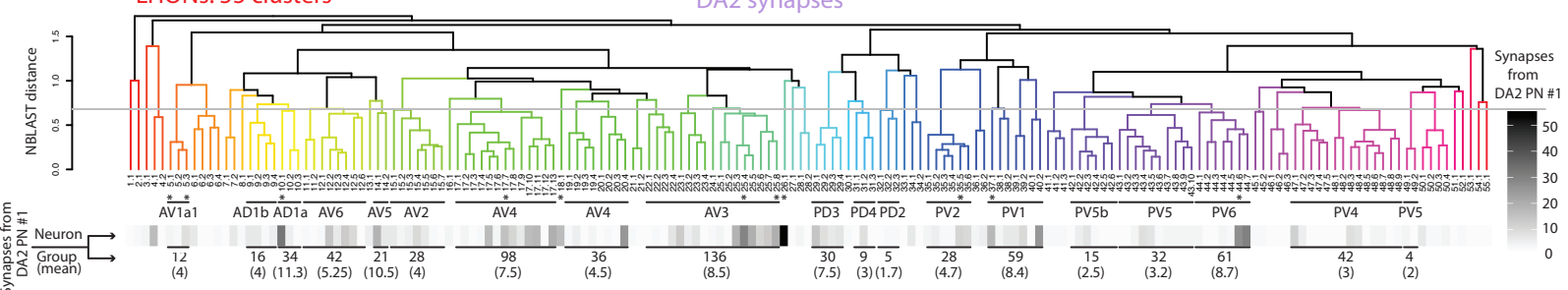

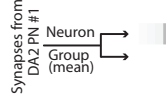

LHLNs: 15 clusters
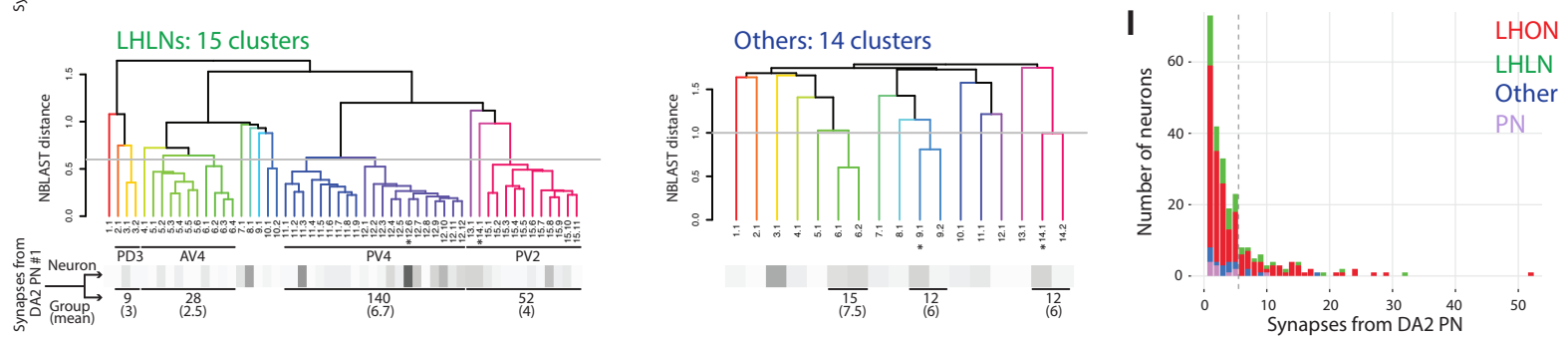

Figure 1: DA2 PNs are necessary for geosmin avoidance, synapse axo-axonically onto the aversive DL4 PNs, and then onto a large number of morphologically diverse third-order neurons. (A) R85E04 labels 5-6 DA2 uniglomerular PNs per hemisphere. (B) Egg-laying two-choice preference indices (PI) to geosmin while silencing DA2 PNs (n=38 for R85E04 and its controls, n=19 for MB247-GAL4/TNT+). (C) Frontal view of DA2 PN axons (green) with examples of other aversive PNs in the LH (DL4, V, DL5). DA4l targets the same area of the LH, but its ligand and valence are unclear. The dashed line marks the putative zone of aversive convergence. (D) An example of a DA2 PN (green) to DL4 PN (orange) axo-axonic synapse in the LH. The arrowheads mark the presynaptic sites. (E) Location of axo-axonic synapses from DA2 to DL4 PNs (open circles). (F) Axo-axonic PN synapses from and to DA2 PNs ( $\mathrm{n}=5$ ) in the right hemisphere. (G1-G4) Downstream targets of DA2 PNs in the LH, by broad neuron class (LHONs, LHLNs, unclassified neurons and PNs). (H) Hierarchical clustering of the DA2 PN downstream target morphologies. Grey lines mark the cut off heights (LHON:0.68, LHLN:0.60, Others:1.0), greyscale bar under the dendrograms the number of synapses from the completed DA2 PN, and asterisks the completed neurons. (I) Distribution of the synaptic connections from the DA2 PN to its downstream targets, color-coded by broad neuron class. Grey line marks the shoulder of the distribution, between 5 and 6 synapses. Significance values: ${ }^{*} \mathrm{p}<0.05^{* *} \mathrm{p}<0.01$ 
by downstream neurons in the $\mathrm{LH}(9,10)$.

To look for downstream targets of DA2 PNs, and thus possible valence integrator neurons, we took two parallel, complementary approaches. First, we reconstructed DA2 PNs and their postsynaptic partners in the LH in a recently acquired whole-brain electron microscopy (EM) volume of a female Drosophila (11), which we refer to as FAFB. We were then able to make comparisons with a second recently acquired partial EM volume of a female (referred to as hemibrain) (12).

Second, we performed a light-level in silico anatomical screen to look for genetic driver lines containing LH neurons (LHNs) putatively downstream of DA2 PNs. We will discuss the EM connectomics approach first.

Previous work had already identified all the uniglomerular PNs on the right (R) side of the EM volume (11), including 5 DA2 PNs. We additionally identified 6 DA2 PNs on the left (L) side of the brain, and marked up all their presynaptic sites on both sides (R: 961, L: 959). Surprisingly, we found a relatively large number (R: 25; L: 32) of axo-axonic synapses from the DA2 PNs to the single aversive DL4 PN (Figure 1D, $\mathrm{E}$ and F, Figure S2A and B), mostly clustered on the ventral axonal branches (Figure 1E). These axo-axonic synapses are also present in the hemibrain (R: 31) Figure S1G). In FAFB no other PN receives more than 2 synapses from the DA2 PNs (Figure 1F), while in the hemibrain, only one other PN receives more than 4 synapses (DA4m) Figure S1G). In both volumes DA2 PNs also synapse strongly onto each other (Figure 1F, Figure S2B), and Figure S1G), thus showing within-odour channel divergence and re-convergence that may serve to increase signal detection speed (13). As a large number of PNs pass close to DA2 synapses without receiving input (Figure S2C), this connectivity appears specific, not just proximity based. This is also supported by the absence of connections from DL4 PNs to DA2 PNs. Axo-axonic integration of PN odour channels has not been previously described, although our recent work (14) has found that it is widespread within PN axons. This type of connectivity may be an important mechanism for valence-based integration, ultimately triggering similar behavioural responses to odours of similar significance to the fly.

We next obtained a complete downstream connectome of the LH targets for a single DA2 PN (Figure S2D). We reconstructed the postsynaptic partners sufficiently to enable unambiguous identification, and quantification of the DA2 PN input. This identified a surprisingly large array of 253 neurons: $171 \mathrm{LH}$ output neurons (LHONs) (Figure 1G1), $54 \mathrm{LH}$ local neurons (Figure 1G2), 11 PNs (Figure 1G4) and 17 other neurons (Figure 1G3). These last 17 include large brain spanning neurons; 10 appear neuromodulatory due to the presence of dense core vesicles or because they are labelled by the TH-GAL4 driver in the FlyCircuit database $(15,16)$.

Together, the 225 LHN target neurons make up 18\% of the estimated total of $\sim 1400$ neurons in the LH (17), a very large fraction given that DA2 is just one of 51 olfactory glomeruli $(2 \%)$. The majority $(\sim 70 \%)$ of DA2 synapses were onto LHONs, and $\sim 21 \%$ onto LHLNs, roughly matching the proportion of neurons in each broad class. The LHONs project to various neuropils thought to be involved in multimodal sensory integration (Figure S2E) (18-23). The neurons are of diverse morphologies: hierarchical clustering of each broad class, splits them into 84 clusters (55 LHONs,
15 LHLNs, 14 others), excluding PNs (Figure 1H). Taken together this suggests the wiring logic of the LH is massively more complex than previously thought $(21,24)$ : PNs from a 'labelled line' glomerulus with clear behavioural meaning do not synapse onto just a few postsynaptic targets. However, the distribution of connectivity is skewed; the majority of targets receive only a few inputs (Figure 1I), and for all 5 RH DA2 PNs in both EM volumes 25\% of all DA2 downstream neurons make up more than $50 \%$ of all synaptic output(Figure S2D). Moreover, LHONs in the same morphological clusters have a higher than chance probability of getting similar levels of DA2 input (Figure S2F, see Methods), but the same is not true for LHLNs (and was not tested for the other neurons, which are more structurally diverse). In summary, the geosmin processing pathway that starts as a labelled line shows convergence at the level of PN axons with another aversive pathway (DL4 PNs), as well as considerable divergence at the transition from second to third-order level.

In order to answer whether valence-based integration takes place in the LH we selected a sample of 15 DA2 strong downstream neurons of diverse morphologies for complete reconstruction in FAFB. As all the uniglomerular excitatory PNs on the right hemisphere were completed we could identify every PN input onto these neurons. Figure $2 \mathrm{~A}$ shows the morphologies of the completed neurons (cell typing according to (14)). We observe a range of input tuning profiles, from DA2 specific (AVLP594, a neuropeptidergic brain-spanning neuron, Figure S2G and H (25)) to completely or nearly aversive odour specific (LHPV6a3\#1, LHAV3a1_c\#1), to relatively broadly tuned (LHAV3f1, LHAV1a1) (Figure 2B). Altogether, 9/15 neurons receive above chance amounts of aversive input from PNs besides DA2. We then identified the same cell types in the hemibrain for comparison. Averaging within cell types and normalising inputs shows few major differences in connectivity for our DA2 downstream targets (Figure 2B). Similar to FAFB, 8/15 neurons in the hemibrain receive above chance amounts of aversive input from non-DA2 PNs.

In addition, most of these neurons receive input from thermo- and hygrosensory VP glomeruli (Figure 2B) (26). This may reflect either direct multisensory integration of aversive signals for extremes of temperature or humidity (critical dangers for insects), or cross-sensory modulation of olfactory pathways by environmental context. These patterns of synaptic connectivity therefore support valence-based integration occurring in the LH. However this is unlikely to be the only computation taking place at this transition from second to third-order level of the circuit.

In parallel with our EM work, we carried out a light-level screen for DA2 downstream neurons and driver lines. We used a registered confocal stack of R85E04 and converted the LH axon arbour into a binary mask (Figure 3A). This allowed us to identify sparse driver lines from the GMR-GAL4 (5) and LH-Split collections (36) containing LHNs with dendrites overlapping the DA2 axons (Figure 3B and C). This in silico anatomical screen identified 18 LHN types, 12 of which could be accessed relatively specifically through either GAL4 or Split-GAL4 lines.

With these reagents in hand, we carried out an optogenetic activation screen hoping to identify aversion triggering LHNs. A total of 27 driver lines (for 12 LHN types) were tested using a four-field arena (Figure 3D, see (36) for full details of 
bioRxiv preprint doi: https://doi.org/10.1101/394403; this version posted November 4, 2020. The copyright holder for this preprint (which was not certified by peer review) is the author/funder, who has granted bioRxiv a license to display the preprint in perpetuity. It is made available under aCC-BY-NC 4.0 International license.

A


AV2d1\#1
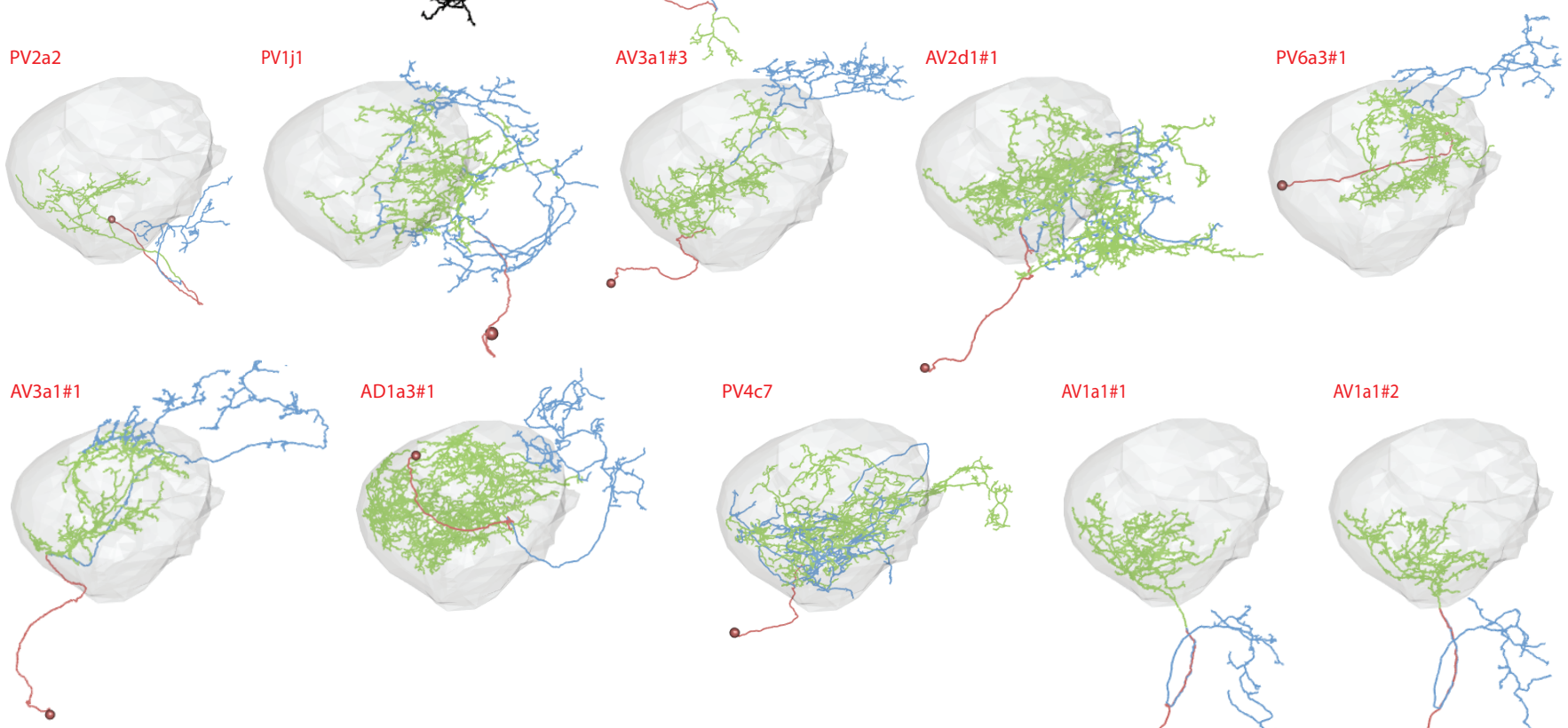

PV4c7

AV1a1\#1

AV1a1\#2

B
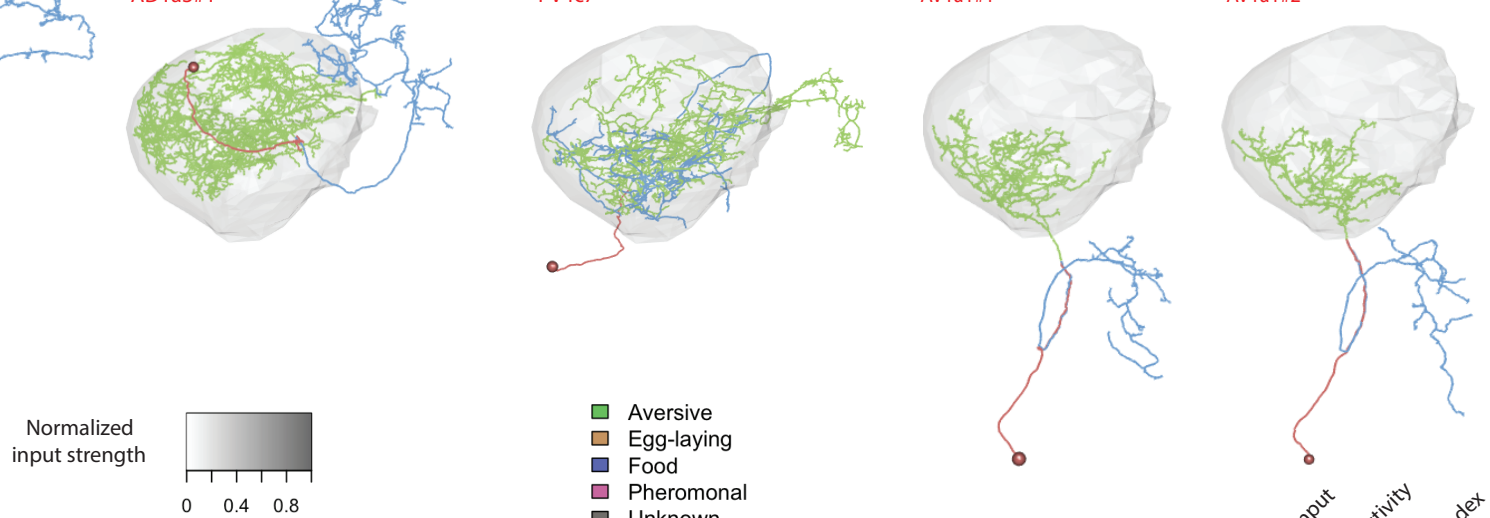

ㅁ Egg-laying

$\square$ Food

$\square$ Pheromona

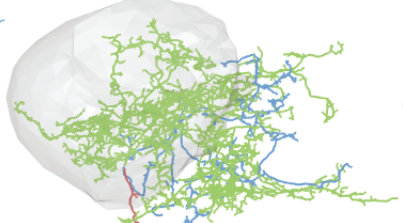

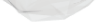

$\square$ Unknown



Figure 2: Strong DA2 downstream targets have diverse tuning breadths but tend to receive more than a chance amount of non-DA2 aversive PN input. (A) EM-reconstructed morphologies of selected strong DA2 targets. Cell bodies and primary neurites are coloured in red, dendrites in green, and axons in blue, where polarity is clear. Neuron names are color-coded by broad neuron class (other: dark blue, LHON: red, LHLN: green). (B) A heatmap representation of excitatory uPN to LHN connectivity for top DA2 targets in both EM volumes, FAFB and the hemibrain, normalised by the total uPN inputs to each neuron, with glomeruli color-coded by putative behavioural relevance $((2,9,27-35))$. The total amount of DA2 input, DA2 selectivity (DA2 input/total uPN input), and Aversion index (DA2 input/uPN input from PN channels with known valence). Neurons receiving more than chance amount of non-DA2 aversive input are marked with an asterisk. 
bioRxiv preprint doi: https://doi.org/10.1101/394403; this version posted November 4, 2020. The copyright holder for this preprint (which was not certified by peer review) is the author/funder, who has granted bioRxiv a license to display the preprint in perpetuity. It is made available under aCC-BY-NC 4.0 International license.

In silico

anatomy screen

of 351 GMR lines:

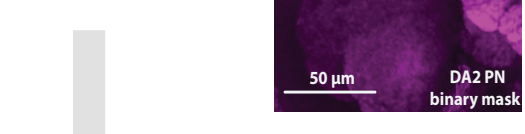

D

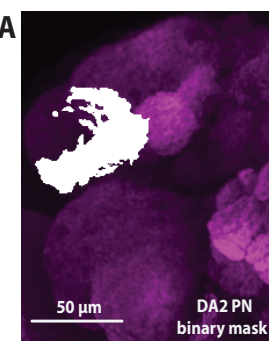

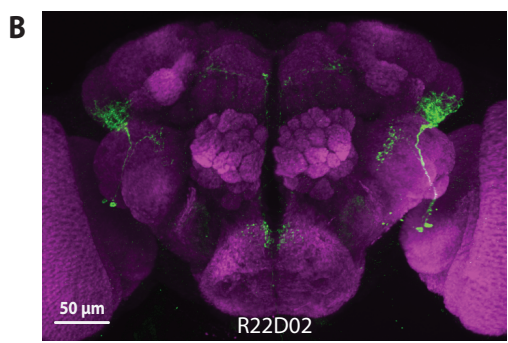

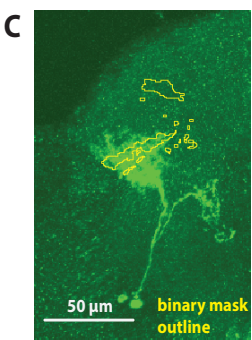

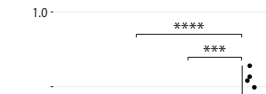


and Split-GAL4
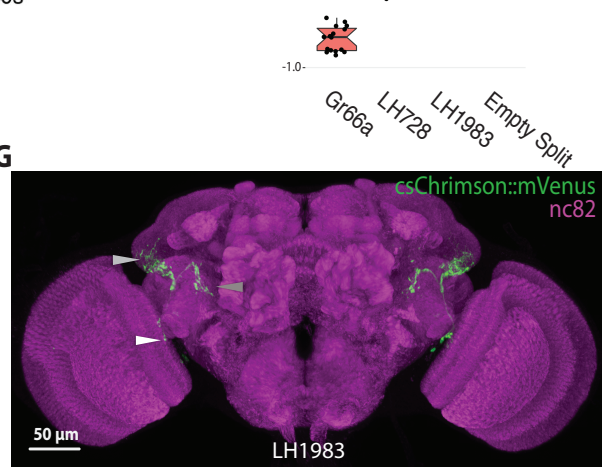

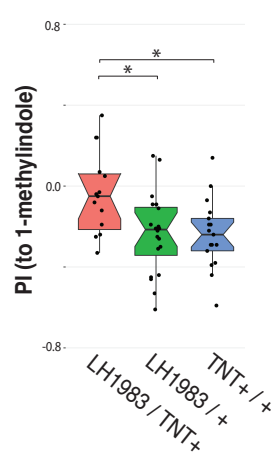

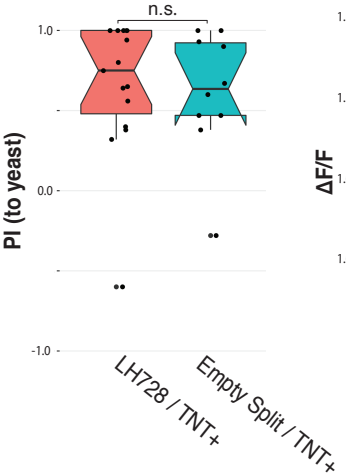

M

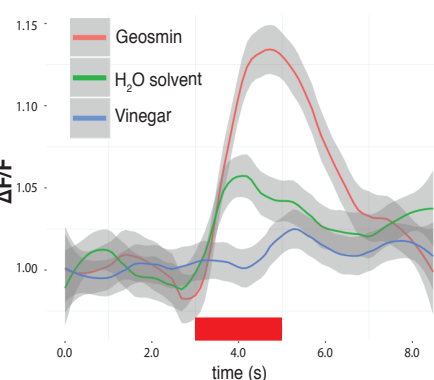

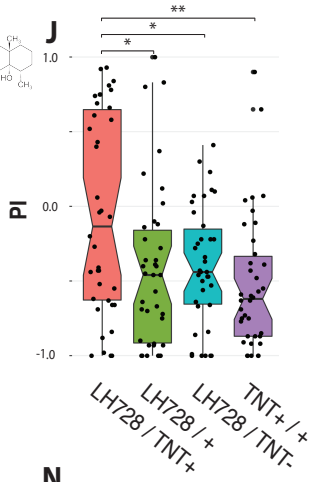

$\mathbf{N}$

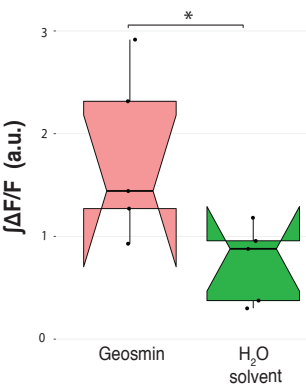

Figure 3: LHAVlal neurons as sufficient for aversion and necessary for both geosmin and 1-methylindole avoidance. (A) LH close-up of the binary mask of DA2 PNs. (B) An example of a good hit in the in silico screen. (C) An example of the binary mask (as an ROI, in yellow) together with a good hit. (D) A schematic representation of the optogenetic four quadrant assay ((37)) used for behavioural screening. 20 female flies explore a circular arena for 30 seconds before two of the quadrants are illuminated with red light for 30 seconds, after which the protocol is repeated by illuminating the remaining two quadrants. Reproduced with permission from ((37)). (E) PI (to the light quadrants) for the last 5 seconds of the stimulation epochs ( $\mathrm{n}=16$, for all groups). (F-G) Expression patterns of LH728 and LH1983. The arrowheads mark the cell bodies (white), dendrites (light grey), and axons (dark grey) of the type LHAVlal LHNs. (H) A 3D rendering of EM reconstructions of LHAVlal\#1 and \#2 (black) overlaid with the LH1983 expression pattern (green). (I) A light-level tracing of the LHAVlal LHNs (black) overlaid with EM-level tracings of LHAVlal\#1 and \#2 (green and magenta, respectively). (J) Egg-laying two-choice PI to geosmin while silencing LHAV1a1 LHNs ( $\mathrm{n}=38$ for all groups). (K) Egg-laying two-choice PI to 1-methylindole in the split-plate assay while silencing LHAV1al LHNs (n=15-20). (L) Egg-laying two-choice PI to yeast odour while silencing LHAV1a1 LHNs (n=14 for both). (M) Odour-evoked in vivo two-photon imaged calcium responses (GCaMP3.0) from LH dendrites of LHAV1al neurons ( $\mathrm{n}=5)$. Red bar marks the stimulation epoch. $(\mathrm{N})$ Area under curve for the stimulation epoch for geosmin and its solvent control (green). Data same as $\mathrm{M}$ ( $\mathrm{n}=5$ ). Significance values: ${ }^{*} \mathrm{p}<0.05^{* *} \mathrm{p}<0.01^{* * *} \mathrm{p}<0.001^{* * * *} \mathrm{p}<0.0001$ 
183 the screen and (37) for the apparatus). Only two of the tested driver lines triggered aversion in this assay (Figure 3E and Figure S3A, B and C): LH728 and LH1983 (Figure 3F and G). Both lines share a parental line (R76E07) and label $\sim 10$ neurons, with somas ventral and medial to the anterior ventrolateral protocerebrum (AVLP). Interestingly, both lines also contain the same two neurons with dendrites in the (ventral) LH and axons in the AVLP, and no other LHNs. The neurons are cholinergic (36), and appear morphologically very similar to the LHAVlal neurons found downstream of DA2 PNs in the EM volume (Figure $3 \mathrm{H}$ ). To confirm this, we generated lightlevel tracings of the neurons in LH1983. As the processes of the two LHNs were in many places too close to resolve, the tracing resulted in a single hybrid skeleton of the two AV1 neurons found in the line. However, overlaying the light-level tracing with LHAVlal\#1 and LHAVlal\#2 reveals a remarkably similar morphology (Figure 3I). Moreover, a quantitative NBLAST (16) comparison to all the 33 neurons taking the $\mathrm{AV} 1$ tract in the EM volume shows that the tops matches are LHAVlal\#1 (similarity score $=0.69$ ) and LHAVlal\#2 (similarity score $=0.65$ ), respectively. Intriguingly, These cells are one of only 3 out of 70 LHN cell types downstream of DA2 PNs that we identified with projections to the ventral rather than superior protocerebrum in the FAFB volume. There are three groups of AVla-like neurons in the hemibrain, belonging to three classes: AVL02q_a_pct ( 3 cells), AVL02q_b_pct (4 cells) and AVL02q_c_pct (2 cells). A comparison of morphology and olfactory inputs shows that AVL02q_c_pct are the most similar to FAFB LHAVlals (Figure 3I and J).

We also tested whether the LHAVlal neurons are necessary for geosmin avoidance by silencing their synaptic activity using tetanus toxin; this abolished geosmin avoidance in the egg-laying assay (Figure 3J). In vivo calcium imaging confirmed that the neurons respond to geosmin, but not to vinegar (a broadly coded attractive odorant) (Figure 3M and $\mathrm{N})$. Taking these functional data together with the fact that no other AV1 tract neurons receive a significant amount of DA2 PN synaptic input in EM data, strongly suggests that the LHAVlal LHNs labelled by both driver lines are necessary and sufficient for some forms of odour avoidance.

Are the other 69 classes of lateral horn neurons that receive geosmin information then irrelevant to its processing? There are a number of possible explanations that could explain this large number of circuit elements.

The input tuning of the pair of LHAVlal neurons that we reconstructed is relatively broad, receiving input from multiple aversive PN channels (including DA2 and DL4). We therefore wondered if these neurons have a general role in aversive odour processing. There are relatively few low concentration repulsive odorants reported in Drosophila. The DL4 ligand iridomyrmecin (9) is not commercially available and pilot control tests with a limited amount of synthetic compound (kindly shared by Jerrit Weißfog and Ales Svatos) did not show robust aversion in our egg laying assay (data not shown). We therefore tested another compound, 1-methylindole, a derivative of the bacterial metabolite indole (38), which we found to be aversive. Silencing LHAVlal neurons also abolished this egg-laying aversion (Figure 3K, see also Figure S3DF, and methods). Indoles are detected by a phylogenetically closely related receptor Or43a $(39,40)$, which projects to the DA4l glomerulus (40). Intriguingly LHAV1a1 LHONs also re- ceive strong input from DA4l PNs (Figure 2B). Importantly, flies were still attracted to yeast odour (Figure 3L), showing they are not anosmic or otherwise unable to respond to odours. Together these data provide evidence for functional integration of aversive odour channels within LHAVlal neurons, which appear to have a selective role in odour avoidance during egg-laying.

Combining functional and connectomics approaches, provides an almost unique opportunity to explore the brainwide logic of olfactory processing. However, the considerable divergence of the geosmin processing pathway when moving from second to third-order neurons, means that it is presently impossible to follow all of the connected LHNs deeper into the brain. Instead we focussed on two cell types that appeared of special interest from the results so far: LHAV1a1 and PV6a3 (an ideal example of an aversive integrator based on its PN inputs).

We identified 14 postsynaptic partners receiving 2 or more synapses downstream of PV6a3, the strongest of which appears to be a neuron projecting to the suboesophageal zone (SEZ) (Fig S4A, B and C). However, as we did not perform an exhaustive reconstruction of the postsynaptic partners, there most likely are more than shown here. Nevertheless, this suggests that one next computational step in the circuit is to integrate aversive odour signals with gustatory ones in the SEZ.

Downstream sampling from the LHAVlal\#1 axon identified 44 postsynaptic partners, many shared with its sibling LHAVlal\#2 (Figure 4A, Figure S4D and E). Downstream sampling in both connectomes showed that LHAVlals share many postsynaptic partners, and, most notably, are all strongly connected to descending neurons (DNs) Figure S4C and D), including two previously unreported DNs, which we have named DNp42 and DNp43, projecting to the nerve cord (Figure 4A). LHAVla1 form many more direct connections with DNs that our other strong DA2 targets Figure S4. Another strong target is DNp06. However, while it receives 5.4\% of LHAVlal output, LHAVlal input to this DN comprises just $0.3 \%$ of the total.

We identified three sparse driver lines for DNp42 (Figure 4B-D, and Figure S5A-C). Silencing DNp42 abolishes geosmin avoidance (Figure 4E), reproducing the phenotype seen at every step in this circuit, from sensory input to these descending neurons. We also optogenetically activated these DNs while observing the flies using high-speed videography (FlyPEZ assay (41)). Strikingly, light-activation triggered a consistent backing up phenotype (Figure 4F) and flies would occasionally take-off, similar to what is seen in response to looming visual stimuli (42). In the VNC, DNp42 axons arborise in all three thoracic neuromeres but stay close to the midline innervating the tectulum (an integrative area), avoiding the more lateral leg neuropils. They also innervate the accessory mesothoracic neuromere and ventral association centre, areas receiving sensory input from the wings and legs, respectively (43) (Figure S6A and B). There are no projections to the abdominal ganglion (which directly controls reproductive functions, including egg laying) and no obvious sexual dimorphism in any of these axonal arbours (Figure S6C). This anatomy is consistent with a pre-motor and/or sensorymotor integration function in locomotor behaviour rather than a direct impact on motoneurons or regulation of egg laying. 


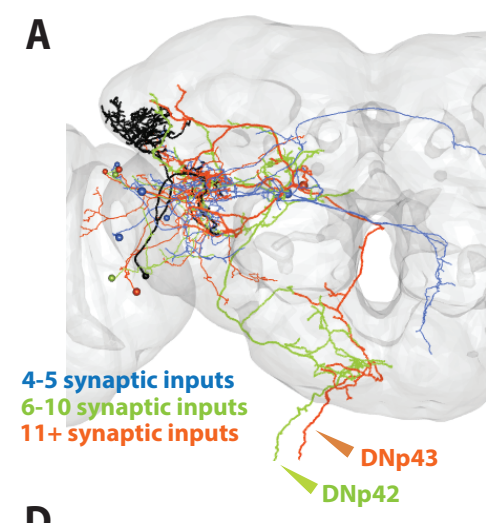

D

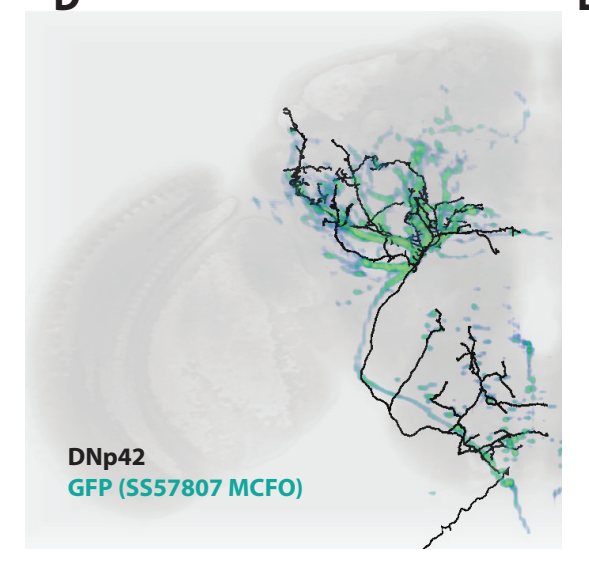

B

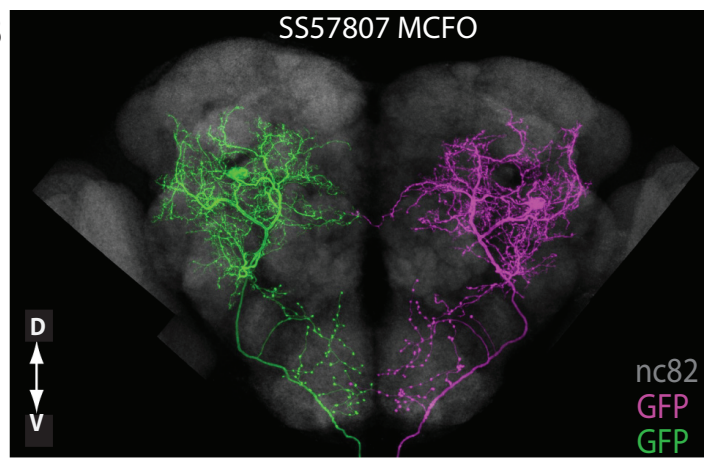

$\mathbf{E}$



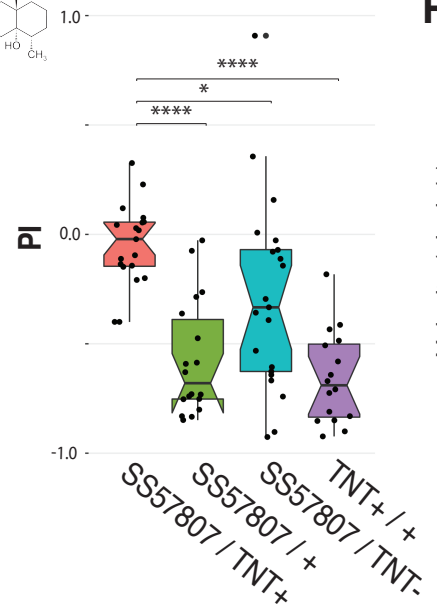

$\mathbf{F}$

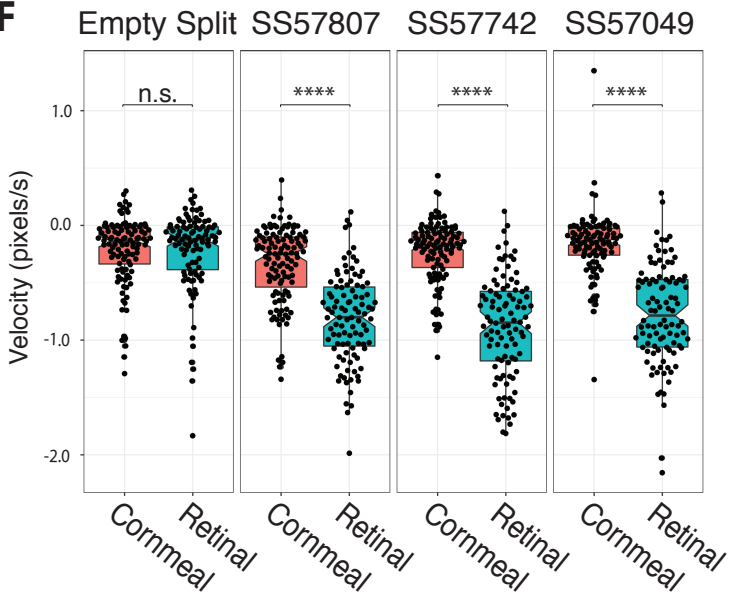

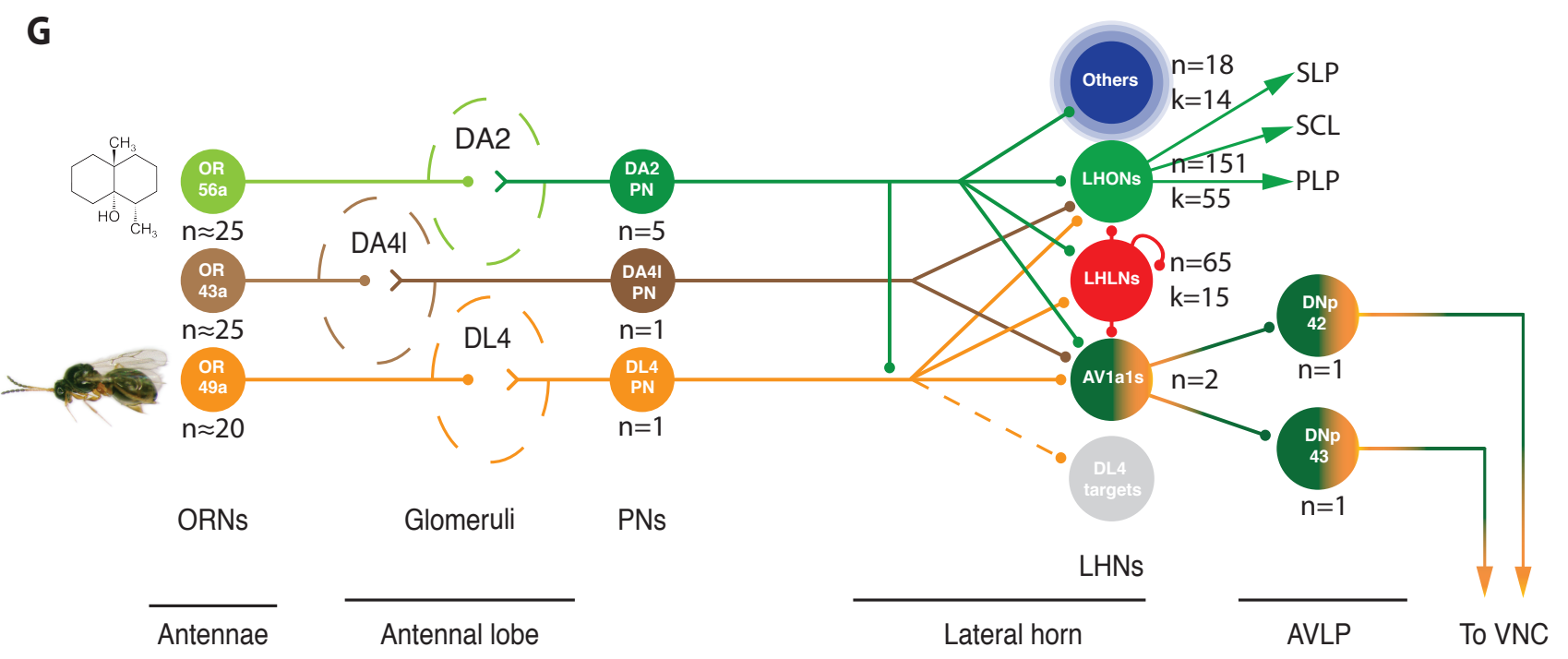

Figure 4: LHAVlal neurons synapse onto DNs that are necessary for geosmin avoidance and sufficient to trigger avoidance behavior. (A) Identified downstream targets of the LHAVlal\#1 with 4 or more synaptic inputs. Neurons are colour-coded according to number of synaptic inputs. Two descending neurons DNp43 (orange) and DNp42 (green) are highlighted with arrowheads. (B-C) MultiColor FlpOut (MCFO) labelling of putative DNp42 neurons in the brain (B) and VNC (C). (D) A 3D rendering of EM reconstruction of DNp42 (black) overlaid with the SS57807 expression pattern (green). (E) Egg-laying two-choice PI to geosmin while silencing DNp42 neurons (n=16-21). (F) Fly velocity in response to optogenetic stimulation of DNp42 neurons via three different driver lines $(\mathrm{n}=99-109)$. Significance values: * $\mathrm{p}<0.05^{* * * *} \mathrm{p}<0.0001$ (G) A simplified schematic of the circuit. Geosmin is detected by Or56a ORNs that project to the AL where they synapse onto DA2 PNs, and local neurons (not shown). The DA2 PNs project to the LH (forming en passant synapses in the MB, not shown). In the LH, DA2 PNs form axo-axonic synapses with parasitic wasp pheromone processing DL4 PNs, and then synapse onto a large number of third-order neurons with varying tuning breadths. LHAVla1 neurons receive input from multiple aversive PN channels (including DL4 and DA4l), and synapse onto DNs that trigger avoidance. Numbers of neurons (n) and clusters (k) are marked. 
While in FAFB there is no exhaustive upstream tracing of DNp42, the autosegmentation of the hemibrain allows us to see it's other inputs. LHAVlals comprise $~ 1.3 \%$ of all input to DNp42. The second strongest input to DNp42, comprising $\sim 2.6 \%$ of all input, is a GABAergic mushroom body output neuron, MBON20(y1y2). This cell arborises in the y1 and y2 lobes of the ventral accessory calyx of the mushroom body. These lobes receive axonal input from protocerebral posterior lateral dopaminergic neurons, which carry predominantly aversive signals. This connectivity resembles the circuit for learned-innate interaction described in (44), though at the level of DNs, rather than LHNs, and with the opposite valence, possibly providing a mechanism by which learned and innate olfactory information is integrated to produce appropriate behavioural responses.

All animals need to solve similar challenges to survive and reproduce in a complex environment. Avoiding pathogens and parasites are some of the ubiquitous ones. Here we trace a microbial-odour processing pathway from sensory neurons all the way through the brain to descending neurons innervating the fly's homologue of the spinal cord (see Figure $4 \mathrm{G}$, for a summary of the circuit). We have identified circuit motifs including multiple levels of valence specific convergence, including clear evidence for convergence of aversive PNs onto common targets, which tend to be co-located in space. This supports the idea of valence-based topography as one organising principle of the LH $(8,10,45)$. More surprisingly, we also found that central olfactory layers show a highly diverging organisation, even for stimuli that are initially coded in a labelled line fashion. This contrasts with our functional data: we trace a single linear pathway essential for aversive egg-laying behaviour, as supported by neuronal activation and/or silencing experiments at each of the 4 layers traversing the brain. Although our analysis of fourth order neurons is far from exhaustive, we have identified few other direct connections to a descending neuron. Therefore this synaptic pathway is probably unusually shallow. This in turn may imply both a particular biological significance and make these neurons more sensitive to simple experimental activation or silencing experiments.

Comparing our connectomics and experimental results challenges us to think about what connection strengths are behaviorally relevant. (46) has recently suggested a classification of major pathways, with 100 or more inputs, and minor pathways, with fewer than about 10. Summing inputs from all 5 DA2 PNs we identified just three target neurons meeting this criterion (range 107-176 connections), not including the LHAV1als, which are individually only the 42nd and 90th strongest DA2 targets by synapse number. Normalising by the total number of inputs, in the majority of cases DA2 PNs accounted for $<5 \%$ of the input to target neurons, and just $1.6 \%$ of the input to the reconstructed LHAV1al neurons. Thus, while absolute thresholds are a useful rule of thumb, it is likely that different criteria will be necessary in different brain areas and between different kinds of neurons. This will be of major significance in interpreting the dense whole brain connectomics datasets in Drosophila recently released (12), with larger brains likely following within the space of a few years (47).

\section{References}

[1] Mark Schaller. The behavioural immune system and the psychology of human sociality. Philos. Trans. R. Soc. Lond. B Biol. Sci., 366(1583):3418-3426, December 2011.

[2] Marcus C Stensmyr, Hany K M Dweck, Abu Farhan, Irene Ibba, Antonia Strutz, Latha Mukunda, Jeanine Linz, Veit Grabe, Kathrin Steck, Sofia Lavista-Llanos, Dieter Wicher, Silke Sachse, Markus Knaden, Paul G Becher, Yoichi Seki, and Bill S Hansson. A conserved dedicated olfactory circuit for detecting harmful microbes in Drosophila. Cell, 151(6):1345-1357, December 2012.

[3] Veit Grabe, Amelie Baschwitz, Hany K M Dweck, Sofia Lavista-Llanos, Bill S Hansson, and Silke Sachse. Elucidating the neuronal architecture of olfactory glomeruli in the Drosophila antennal lobe. Cell Rep., 16(12):34013413, September 2016.

[4] Nicolas Y Masse, Glenn C Turner, and Gregory S X E Jefferis. Olfactory information processing in Drosophila. Current Biology, 19(16):R700-R713, 2009.

[5] Arnim Jenett, Gerald M Rubin, Teri-T B Ngo, David Shepherd, Christine Murphy, Heather Dionne, Barret D Pfeiffer, Amanda Cavallaro, Donald Hall, Jennifer Jeter, Nirmala Iyer, Dona Fetter, Joanna H Hausenfluck, Hanchuan Peng, Eric T Trautman, Robert R Svirskas, Eugene W Myers, Zbigniew R Iwinski, Yoshinori Aso, Gina M DePasquale, Adrianne Enos, Phuson Hulamm, Shing Chun Benny Lam, Hsing-Hsi Li, Todd R Laverty, Fuhui Long, Lei Qu, Sean D Murphy, Konrad Rokicki, Todd Safford, Kshiti Shaw, Julie H Simpson, Allison Sowell, Susana Tae, Yang Yu, and Christopher T Zugates. A GAL4-driver line resource for Drosophila neurobiology. Cell Rep., 2(4):991-1001, October 2012.

[6] Johannes Kohl, Julian Ng, Sebastian Cachero, Ernesto Ciabatti, Michael-John Dolan, Ben Sutcliffe, Adam Tozer, Sabine Ruehle, Daniel Krueger, Shahar Frechter, Tiago Branco, Marco Tripodi, and Gregory S X E Jefferis. Ultrafast tissue staining with chemical tags. Proc. Natl. Acad. Sci. U. S. A., 111(36):E3805-14, September 2014.

[7] Sean T Sweeney, Kendal Broadie, John Keane, Heiner Niemann, and Cahir J O'Kane. Targeted expression of tetanus toxin light chain in Drosophila specifically eliminates synaptic transmission and causes behavioral defects. Neuron, 14(2):341-351, 1995.

[8] Sonia G Chin, Sarah E Maguire, Paavo Huoviala, Gregory S X E Jefferis, and Christopher J Potter. Olfactory neurons and brain centers directing oviposition decisions in Drosophila. Cell Rep., 24(6):1667-1678, August 2018.

[9] Shimaa A M Ebrahim, Hany K M Dweck, Johannes Stökl, John E Hofferberth, Federica Trona, Kerstin Weniger, Jürgen Rybak, Yoichi Seki, Marcus C Stensmyr, Silke Sachse, Bill S Hansson, and Markus Knaden. Drosophila avoids parasitoids by sensing their semiochemicals via a dedicated olfactory circuit. PLoS Biol., 13(12):e1002318, December 2015. 
[10] Gregory S X E Jefferis, Christopher J Potter, Alexander M Chan, Elizabeth C Marin, Torsten Rohlfing, Calvin R Maurer, Jr, and Liqun Luo. Comprehensive maps of Drosophila higher olfactory centers: spatially segregated fruit and pheromone representation. Cell, 128(6): 1187-1203, March 2007.

[11] Zhihao Zheng, J Scott Lauritzen, Eric Perlman, Camenzind G Robinson, Matthew Nichols, Daniel Milkie, Omar Torrens, John Price, Corey B Fisher, Nadiya Sharifi, Steven A Calle-Schuler, Lucia Kmecova, Iqbal J Ali, Bill Karsh, Eric T Trautman, John A Bogovic, Philipp Hanslovsky, Gregory S X E Jefferis, Michael Kazhdan, Khaled Khairy, Stephan Saalfeld, Richard D Fetter, and Davi D Bock. A complete electron microscopy volume of the brain of adult Drosophila melanogaster. Cell, 174 (3):730-743.e22, July 2018.

[12] Louis K Scheffer, C Shan Xu, Michal Januszewski, Zhiyuan Lu, Shin-ya Takemura, Kenneth J Hayworth, Gary B Huang, Kazunori Shinomiya, Jeremy MaitlinShepard, Stuart Berg, Jody Clements, Philip M Hubbard, William T Katz, Lowell Umayam, Ting Zhao, David Ackerman, Tim Blakely, John Bogovic, Tom Dolafi, Dagmar Kainmueller, Takashi Kawase, Khaled A Khairy, Laramie Leavitt, Peter H Li, Larry Lindsey, Nicole Neubarth, Donald J Olbris, Hideo Otsuna, Eric T Trautman, Masayoshi Ito, Alexander S Bates, Jens Goldammer, Tanya Wolff, Robert Svirskas, Philipp Schlegel, Erika Neace, Christopher J Knecht, Chelsea X Alvarado, Dennis A Bailey, Samantha Ballinger, Jolanta A Borycz, Brandon S Canino, Natasha Cheatham, Michael Cook, Marisa Dreher, Octave Duclos, Bryon Eubanks, Kelli Fairbanks, Samantha Finley, Nora Forknall, Audrey Francis, Gary Patrick Hopkins, Emily M Joyce, SungJin Kim, Nicole A Kirk, Julie Kovalyak, Shirley A Lauchie, Alanna Lohff, Charli Maldonado, Emily A Manley, Sari McLin, Caroline Mooney, Miatta Ndama, Omotara Ogundeyi, Nneoma Okeoma, Christopher Ordish, Nicholas Padilla, Christopher M Patrick, Tyler Paterson, Elliott E Phillips, Emily M Phillips, Neha Rampally, Caitlin Ribeiro, Madelaine K Robertson, Jon Thomson Rymer, Sean M Ryan, Megan Sammons, Anne K Scott, Ashley L Scott, Aya Shinomiya, Claire Smith, Kelsey Smith, Natalie L Smith, Margaret A Sobeski, Alia Suleiman, Jackie Swift, Satoko Takemura, Iris Talebi, Dorota Tarnogorska, Emily Tenshaw, Temour Tokhi, John J Walsh, Tansy Yang, Jane Anne Horne, Feng Li, Ruchi Parekh, Patricia K Rivlin, Vivek Jayaraman, Marta Costa, Gregory SXE Jefferis, Kei Ito, Stephan Saalfeld, Reed George, Ian A Meinertzhagen, Gerald M Rubin, Harald F Hess, Viren Jain, and Stephen M Plaza. A connectome and analysis of the adult Drosophila central brain. eLife, 9:e57443, sep 2020. ISSN 2050-084X. doi: 10.7554/eLife.57443. URL https://doi.org/10.7554/ eLife.57443.

[13] James M Jeanne and Rachel I Wilson. Convergence, divergence, and reconvergence in a feedforward network improves neural speed and accuracy. Neuron, 88(5): 1014-1026, December 2015.
[14] Alexander S. Bates, Philipp Schlegel, Ruairi J.V. Roberts, Nikolas Drummond, Imaan F.M. Tamimi, Robert Turnbull, Xincheng Zhao, Elizabeth C. Marin, Patricia D. Popovici, Serene Dhawan, Arian Jamasb, Alexandre Javier, Laia Serratosa Capdevila, Feng Li, Gerald M. Rubin, Scott Waddell, Davi D. Bock, Marta Costa, and Gregory S.X.E. Jefferis. Complete connectomic reconstruction of olfactory projection neurons in the fly brain. Current Biology, 30(16):3183 - 3199.e6, 2020. ISSN 0960-9822. doi: https://doi.org/10.1016/j.cub.2020. 06.042. URL http://www.sciencedirect.com/science/ article/pii/S0960982220308587.

[15] Ann-Shyn Chiang, Chih-Yung Lin, Chao-Chun Chuang, Hsiu-Ming Chang, Chang-Huain Hsieh, Chang-Wei Yeh, Chi-Tin Shih, Jian-Jheng Wu, Guo-Tzau Wang, YungChang Chen, Cheng-Chi Wu, Guan-Yu Chen, Yu-Tai Ching, Ping-Chang Lee, Chih-Yang Lin, Hui-Hao Lin, Chia-Chou Wu, Hao-Wei Hsu, Yun-Ann Huang, JingYi Chen, Hsin-Jung Chiang, Chun-Fang Lu, Ru-Fen $\mathrm{Ni}$, Chao-Yuan Yeh, and Jenn-Kang Hwang. Threedimensional reconstruction of brain-wide wiring networks in Drosophila at single-cell resolution. Curr. Biol., 21(1):1-11, January 2011.

[16] Marta Costa, James D Manton, Aaron D Ostrovsky, Steffen Prohaska, and Gregory S X E Jefferis. NBLAST: Rapid, sensitive comparison of neuronal structure and construction of neuron family databases. Neuron, 91(2): 293-311, July 2016.

[17] Shahar Frechter, Alexander Shakeel Bates, Sina Tootoonian, Michael-John Dolan, James Manton, Arian Rokkum Jamasb, Johannes Kohl, Davi Bock, and Gregory Jefferis. Functional and anatomical specificity in a higher olfactory centre. eLife, 8:e44590, may 2019. ISSN 2050-084X. doi: 10.7554/eLife.44590. URL https://doi.org/10.7554/eLife.44590.

[18] Kei Ito, Kazunori Shinomiya, Masayoshi Ito, J Douglas Armstrong, George Boyan, Volker Hartenstein, Steffen Harzsch, Martin Heisenberg, Uwe Homberg, Arnim Jenett, Haig Keshishian, Linda L Restifo, Wolfgang Rössler, Julie H Simpson, Nicholas J Strausfeld, Roland Strauss, Leslie B Vosshall, and Insect Brain Name Working Group. A systematic nomenclature for the insect brain. Neuron, 81(4):755-765, February 2014.

[19] Azusa Kamikouchi, Takashi Shimada, and Kei Ito. Comprehensive classification of the auditory sensory projections in the brain of the fruit fly Drosophila melanogaster. J. Comp. Neurol., 499(3):317-356, 2006.

[20] Tetsuya Miyamoto and Hubert Amrein. Suppression of male courtship by a Drosophila pheromone receptor. Nat. Neurosci., 11(8):874-876, August 2008.

[21] Vanessa Ruta, Sandeep Robert Datta, Maria Luisa Vasconcelos, Jessica Freeland, Loren L Looger, and Richard Axel. A dimorphic pheromone circuit in Drosophila from sensory input to descending output. Nature, 468 (7324):686-690, 2010. 
bioRxiv preprint doi: https://doi.org/10.1101/394403; this version posted November 4, 2020. The copyright holder for this preprint (which was not certified by peer review) is the author/funder, who has granted bioRxiv a license to display the preprint in perpetuity. It is made available under aCC-BY-NC 4.0 International license.

22] E Josephine Clowney, Shinya Iguchi, Jennifer J Bussell, Elias Scheer, and Vanessa Ruta. Multimodal chemosensory circuits controlling male courtship in Drosophila. Neuron, 87(5):1036-1049, September 2015.

[23] Chi-Tin Shih, Olaf Sporns, Shou-Li Yuan, Ta-Shun Su, Yen-Jen Lin, Chao-Chun Chuang, Ting-Yuan Wang, Chung-Chuang Lo, Ralph J Greenspan, and Ann-Shyn Chiang. Connectomics-based analysis of information flow in the Drosophila brain. Curr. Biol., 25(10):12491258, May 2015.

[24] Johannes Kohl, Aaron D Ostrovsky, Shahar Frechter, and Gregory S X E Jefferis. A bidirectional circuit switch reroutes pheromone signals in male and female brains. Cell, 155(7):1610-1623, December 2013.

[25] Hongbo Jiang, Ankhbayar Lkhagva, Ivana Daubnerová, Hyo-Seok Chae, Ladislav Šimo, Sung-Hwan Jung, YeuKyung Yoon, Na-Rae Lee, Jae Young Seong, Dušan Žitňan, Yoonseong Park, and Young-Joon Kim. Natalisin, a tachykinin-like signaling system, regulates sexual activity and fecundity in insects. Proc. Natl. Acad. Sci. U. S. A., 110(37):E3526-34, September 2013.

[26] Elizabeth C. Marin, Laurin Büld, Maria Theiss, Tatevik Sarkissian, Ruairí J.V. Roberts, Robert Turnbull, Imaan F.M. Tamimi, Markus W. Pleijzier, Willem J. Laursen, Nik Drummond, Philipp Schlegel, Alexander S. Bates, Feng Li, Matthias Landgraf, Marta Costa, Davi D. Bock, Paul A. Garrity, and Gregory S.X.E. Jefferis. Connectomics analysis reveals first-, second-, and thirdorder thermosensory and hygrosensory neurons in the adult drosophila brain. Current Biology, 30(16):3167 3182.e4, 2020. ISSN 0960-9822. doi: https://doi.org/10. 1016/j.cub.2020.06.028. URL http://www.sciencedirect. com/science/article/pii/S0960982220308447.

[27] Minrong Ai, Soohong Min, Yael Grosjean, Charlotte Leblanc, Rati Bell, Richard Benton, and Greg S B Suh. Acid sensing by the Drosophila olfactory system. $\mathrm{Na}$ ture, 468(7324):691-695, 2010.

[28] Markus Knaden, Antonia Strutz, Jawaid Ahsan, Silke Sachse, and Bill S Hansson. Spatial representation of odorant valence in an insect brain. Cell Rep., 1(4):392399, 2012.

[29] Julia L Semmelhack and Jing W Wang. Select Drosophila glomeruli mediate innate olfactory attraction and aversion. Nature, 459(7244):218-223, May 2009.

[30] Greg S B Suh, Allan M Wong, Anne C Hergarden, Jing W Wang, Anne F Simon, Seymour Benzer, Richard Axel, and David J Anderson. A single population of olfactory sensory neurons mediates an innate avoidance behaviour in Drosophila. Nature, 431(7010):854-859, October 2004.

[31] Suzan Mansourian, Jacob Corcoran, Anders Enjin, Christer Löfstedt, Marie Dacke, and Marcus C Stensmyr. Fecal-Derived phenol induces Egg-Laying aversion in Drosophila. Curr. Biol., 26(20):2762-2769, October 2016.
[32] Suzan Mansourian and Marcus C Stensmyr. The chemical ecology of the fly. Curr. Opin. Neurobiol., 34:95-102, October 2015.

[33] Daniel Münch and C Giovanni Galizia. DoOR 2.0 - comprehensive mapping of Drosophila melanogaster odorant responses. Sci. Rep., 6(1), 2016.

[34] Amina Kurtovic, Alexandre Widmer, and Barry J Dickson. A single class of olfactory neurons mediates behavioural responses to a Drosophila sex pheromone. Nature, 446(7135):542-546, March 2007.

[35] Johannes Kohl, Paavo Huoviala, and Gregory Sxe Jefferis. Pheromone processing in Drosophila. Curr. Opin. Neurobiol., 34:149-157, October 2015.

[36] Michael-John Dolan, Shahar Frechter, Alexander Shakeel Bates, Chuntao Dan, Paavo Huoviala, Ruairí Jv Roberts, Philipp Schlegel, Serene Dhawan, Remy Tabano, Heather Dionne, Christina Christoforou, Kari Close, Ben Sutcliffe, Bianca Giuliani, Feng Li, Marta Costa, Gudrun Ihrke, Geoffrey Wilson Meissner, Davi D Bock, Yoshinori Aso, Gerald M Rubin, and Gregory Sxe Jefferis. Neurogenetic dissection of the lateral horn reveals major outputs, diverse behavioural functions, and interactions with the mushroom body. Elife, 8, May 2019.

[37] Yoshinori Aso, Divya Sitaraman, Toshiharu Ichinose, Karla R Kaun, Katrin Vogt, Ghislain Belliart-Guérin, Pierre-Yves Plaçais, Alice A Robie, Nobuhiro Yamagata, Christopher Schnaitmann, William J Rowell, Rebecca M Johnston, Teri-T B Ngo, Nan Chen, Wyatt Korff, Michael N Nitabach, Ulrike Heberlein, Thomas Preat, Kristin M Branson, Hiromu Tanimoto, and Gerald M Rubin. Mushroom body output neurons encode valence and guide memory-based action selection in Drosophila. Elife, 3:e04580, December 2014.

[38] Jin-Hyung Lee and Jintae Lee. Indole as an intercellular signal in microbial communities. FEMS Microbiol. Rev., 34(4):426-444, July 2010.

[39] Ian W Keesey, Jin Zhang, Ana Depetris-Chauvin, George F Obiero, Markus Knaden, and Bill S Hansson. Evolution of a pest: towards the complete neuroethology of Drosophila suzukii and the subgenus sophophora. bioRxiv, July 2019.

[40] Africa Couto, Mattias Alenius, and Barry J Dickson. Molecular, anatomical, and functional organization of the Drosophila olfactory system. Curr. Biol., 15(17): 1535-1547, September 2005.

[41] W Ryan Williamson, Martin Y Peek, Patrick Breads, Brian Coop, and Gwyneth M Card. Tools for rapid High-Resolution behavioral phenotyping of automatically isolated Drosophila. Cell Rep., 25(6):1636-1649.e5, November 2018.

[42] Ming Wu, Aljoscha Nern, W Ryan Williamson, Mai M Morimoto, Michael B Reiser, Gwyneth M Card, and Gerald M Rubin. Visual projection neurons in the lobula 
bioRxiv preprint doi: https://doi.org/10.1101/394403; this version posted November 4, 2020. The copyright holder for this preprint (which was not certified by peer review) is the author/funder, who has granted bioRxiv a license to display the preprint in perpetuity. It is made available under aCC-BY-NC 4.0 International license.

link feature detection to distinct behavioral programs. Elife, 5, December 2016.

[43] Lalanti Venkatasubramanian and Richard S Mann. The development and assembly of the Drosophila adult ventral nerve cord. Curr. Opin. Neurobiol., 56:135-143, June 2019.

[44] Michael-John Dolan, Ghislain Belliart-Guérin, Alexander Shakeel Bates, Shahar Frechter, Aurélie LampinSaint-Amaux, Yoshinori Aso, Ruairí JV Roberts, Philipp Schlegel, Allan Wong, Adnan Hammad, et al. Communication from learned to innate olfactory processing centers is required for memory retrieval in drosophila. Neuron, 100(3):651-668, 2018.

[45] Antonia Strutz, Jan Soelter, Amelie Baschwitz, Abu Farhan, Veit Grabe, Jürgen Rybak, Markus Knaden, Michael Schmuker, Bill S Hansson, and Silke Sachse. Decoding odor quality and intensity in the Drosophila brain. Elife, 3:e04147, December 2014.

[46] Ian A Meinertzhagen. Of what use is connectomics? a personal perspective on the connectome. J. Exp. Biol., 221(Pt 10), May 2018.

[47] Louis K Scheffer and Ian A Meinertzhagen. The fly brain atlas. Annu. Rev. Cell Dev. Biol., 35:637-653, October 2019.

[48] Nathan C Klapoetke, Yasunobu Murata, Sung Soo Kim, Stefan R Pulver, Amanda Birdsey-Benson, Yong Ku Cho, Tania K Morimoto, Amy S Chuong, Eric J Carpenter, Zhijian Tian, Jun Wang, Yinlong Xie, Zhixiang Yan, Yong Zhang, Brian Y Chow, Barbara Surek, Michael Melkonian, Vivek Jayaraman, Martha Constantine-Paton, Gane Ka-Shu Wong, and Edward S Boyden. Independent optical excitation of distinct neural populations. Nat. Methods, 11(3):338-346, March 2014.

[49] C Dustin Rubinstein, Patricia K Rivlin, and Ron R Hoy. Genetic feminization of the thoracic nervous system disrupts courtship song in male Drosophila melanogaster. J. Neurogenet., 24(4):234-245, December 2010 .

[50] Haojiang Luan, Nathan C Peabody, Charles R Vinson, and Benjamin H White. Refined spatial manipulation of neuronal function by combinatorial restriction of transgene expression. Neuron, 52(3):425-436, November 2006 .

[51] Shigehiro Namiki, Michael H Dickinson, Allan M Wong, Wyatt Korff, and Gwyneth M Card. The functional organization of descending sensory-motor pathways in. Elife, 7, June 2018.

[52] Heather Dionne, Karen L. Hibbard, Amanda Cavallaro, Jui-Chun Kao, and Gerald M. Rubin. Genetic reagents for making split-gal4 lines in drosophila. Genetics, 209 (1):31-35, 2018. ISSN 0016-6731. doi: 10.1534/genetics. 118.300682. URL https://www.genetics.org/content/ 209/1/31.
[53] Laszlo Tirian and Barry J Dickson. The VT GAL4, LexA, and split-GAL4 driver line collections for targeted expression in the Drosophila nervous system. bioRxiv, October 2017.

[54] Aljoscha Nern, Barret D Pfeiffer, and Gerald M Rubin. Optimized tools for multicolor stochastic labeling reveal diverse stereotyped cell arrangements in the fly visual system. Proc. Natl. Acad. Sci. U. S. A., 112(22):E2967-76, June 2015.

[55] Yoshinori Aso, Daisuke Hattori, Yang Yu, Rebecca M Johnston, Nirmala A Iyer, Teri-Tb Ngo, Heather Dionne, L F Abbott, Richard Axel, Hiromu Tanimoto, and Gerald M Rubin. The neuronal architecture of the mushroom body provides a logic for associative learning. eLife, 3, 2014.

[56] Stephan Saalfeld, Albert Cardona, Volker Hartenstein, and Pavel Tomancak. CATMAID: collaborative annotation toolkit for massive amounts of image data. Bioinformatics, 25(15):1984-1986, August 2009.

[57] Casey M Schneider-Mizell, Stephan Gerhard, Mark Longair, Tom Kazimiers, Feng Li, Maarten F Zwart, Andrew Champion, Frank M Midgley, Richard D Fetter, Stephan Saalfeld, and Albert Cardona. Quantitative neuroanatomy for connectomics in Drosophila. Elife, 5, March 2016.

[58] Nicolas Y Masse, Sebastian Cachero, Aaron D Ostrovsky, and Gregory S X E Jefferis. A mutual information approach to automate identification of neuronal clusters in Drosophila brain images. Front. Neuroinform., 6:21, June 2012.

[59] Alexander Shakeel Bates, James D Manton, Sridhar R Jagannathan, Marta Costa, Philipp Schlegel, Torsten Rohlfing, and Gregory SXE Jefferis. The natverse, a versatile toolbox for combining and analysing neuroanatomical data. eLife, 9:e53350, apr 2020. ISSN 2050084X. doi: 10.7554/eLife.53350. URL https://doi.org/10. 7554/eLife.53350.

[60] Robert Court, Shigehiro Namiki, J. Douglas Armstrong, Jana Börner, Gwyneth Card, Marta Costa, Michael Dickinson, Carsten Duch, Wyatt Korff, Richard Mann, David Merritt, Rod K. Murphey, Andrew M. Seeds, Troy Shirangi, Julie H. Simpson, James W. Truman, John C. Tuthill, Darren W. Williams, and David Shepherd. A systematic nomenclature for the drosophila ventral nerve cord. Neuron, 107(6):1071 - 1079.e2, 2020. ISSN 0896-6273. doi: https://doi.org/10.1016/j.neuron.2020. 08.005. URL http://www.sciencedirect.com/science/ article/pii/S0896627320306127.

[61] Sebastian Cachero, Aaron D Ostrovsky, Jai Y Yu, Barry J Dickson, and Gregory S X E Jefferis. Sexual dimorphism in the fly brain. Curr. Biol., 20(18):1589-1601, September 2010.

[62] Torsten Rohlfing and Calvin R Maurer, Jr. Nonrigid image registration in shared-memory multiprocessor environments with application to brains, breasts, and bees.

\section{1} 722

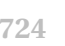
25 726 727 728 
bioRxiv preprint doi: https://doi.org/10.1101/394403; this version posted November 4, 2020. The copyright holder for this preprint (which was not certified by peer review) is the author/funder, who has granted bioRxiv a license to display the preprint in perpetuity. It is made available under aCC-BY-NC 4.0 International license.

IEEE Trans. Inf. Technol. Biomed., 7(1):16-25, March 2003.

[63] Thomas A Pologruto, Bernardo L Sabatini, and Karel Svoboda. ScanImage: flexible software for operating laser scanning microscopes. Biomed. Eng. Online, 2:13, May 2003.

\section{Materials and Methods}

\section{Drosophila stocks, driver line generation, and husbandry}

The following stocks were used: Canton S (UC San Diego Drosophila Stock Center, CA); Ir8a1 ; Ir25a2 ; Orco1 , Gr63a1 (a kind gift from R. Benton); w; Or56a-GAL4; + (Bloomington Drosophila Stock Center, Indiana); w; Or56a-/-; + (a kind gift from Christopher Potter) (8); w; MB247-GAL4; + (Bloomington Drosophila Stock Center, Indiana); w; UAS-Kir2.1::GFP;+ (a kind gift from Matthias Landgraf); w; +; R85E04, Bloomington Drosophila Stock Center, Indiana) (5); w; UAS-TNT-active form; w; UAS-TNT-inactive form (both kind gifts from C.O'Kane) (7); 20XUAS-IVSChrimsonR::mVenus (attP18);+;+ (48); w; UAS-GCaMP3.0 (attP18); UAS-GCaMP3.0 (attP40); w; UAS-mCD8::GFP; UAS-mCD8::GFP; +; y, w; poxnMB00113; + (Bloomington Drosophila Stock Center, Indiana); teashirt-GAL80 (49); ZpGAL4DBD, pJFRC200-10XUASIVS-myr::smGFP-HA (attP18); +; +; p65ADZp(su(Hw) attP8); +; +; w;p65ADZp(su(Hw) attP40); +; w; +; p65ADZp(su(Hw) VK00027); pJFRC513xUAS-Syt::smGFP-HA ((Hw) attP1); pJFRC225-5xUAS-IVSmyr::smGFP-FLAG (VK00005).

The LH Split-GAL4 (50) lines for the optogenetic screen were made as a part of a larger collaborative screen for creating a cell-type specific driver line library for LHNs (details described in (36)), using a subset of the enhancer fragments used in generating the original GMR GAL4 lines (5). The Split-GAL4 lines for the DNs were made essentially similarly to (51). Based on our screening of GAL4 and GAL4 with teashirt lines, we selected AD/DBD combinations from the Janelia (52) and VT (53) collections that we thought shared expression in individual DNs. To visualize combined expression patterns, we crossed males carrying a GFP reporter (pJFRC200-10XUASIVS-myr::smGFP-HA in attP18) and the ZpGAL4DBD transgene (in attP2) with virgin females carrying the p65ADZp transgene in either su(Hw)attP8, attP40, or VK00027 and examined expression in 3- to 10-day-old female progeny. The split-GAL4 combinations that we deemed sparse enough to include in our DN collection were made into stable stocks containing the $\mathrm{AD}$ and $\mathrm{DBD}$ transgenes. To obtain polarity and higher resolution $(40 \mathrm{x}, 63 \mathrm{x})$ information on selected lines, split-GAL4 lines were crossed to pJFRC51-3xUAS-Syt::smGFP-HA in su(Hw)attP1; pJFRC2255xUAS-IVS-myr::smGFP-FLAG in VK00005 and processed for imaging. We used the multicolor flip out technique to stochastically label individual neurons in lines that contained multiple cells (54). These protocols are available on the Janelia FlyLight website (https://www.janelia.org/ project-team/flylight/protocols). Some split-GAL4 lines were also crossed to 20XUAS-CsChrimson-mVenus trafficked in
attP18 (virginator stock) and processed as above to visualize expression pattern when using the CsChrimson effector, as observed expression patterns are known to vary slightly depending on the reporter used (55). Based on their GFP or CsChrimson expression patterns, we made our best estimate of the number of background (non-targeted-DN) cell types in each split-GAL4 line made, and we gave each split line a quality score of A (no background expression), B (one background cell type), or C (two or more background cell types). Confocal image stacks of the stabilized split-GAL4 intersections are available online (http://www.janelia.org/split-gal4). For most experiments flies were reared at $25 \mathrm{C}$ and $60 \% \mathrm{hu}$ midity, under a 12:12 hour light-dark cycle, on food made with the following recipe: $4.81 \mathrm{H} 2 \mathrm{O}, 275 \mathrm{~g}$ of Glucose, 250 g yeast, $37 \mathrm{~g}$ agar, $175 \mathrm{~g}$ flour, $125 \mathrm{ml}$ Nipagen solution, 50 $\mathrm{ml}$ penicillin/streptomycin, $20 \mathrm{ml}$ propionic acid. The same food was also used for the egg-laying assays. For optogenetic behavioural experiments, flies were reared at $22 \mathrm{C}$ on standard Iberian food containing yeast, cornmeal and agar, and supplemented with 1/500 all-trans retinal (Sigma-Aldrich, St. Louis, USA).

\section{Odor stimuli}

Geosmin (CAS \#16423-19-1) was used at a concentration of 1:1000 (Sigma-Aldrich, St. Louis, USA , Product Number UC18). 1-Methylindole (CAS \#603-76-9) was used at either 1:1000 or 1:10.000 concentration (Sigma-Aldrich, St. Louis, USA, Product number 193984). The odors, concentrations, and odor delivery used for electrophysiology and calcium imaging were the same as used in (17).

\section{Egg-laying two-choice assay}

Female flies were collected on the day of eclosion under $\mathrm{CO} 2$ anaesthesia, reared in same sex vials at $25 \mathrm{C}$ and $60 \%$ humidity. Female flies aged 5-7 days were then mated with males of similar age for 6 hours on the day of the experiments. After six hours of mating the female flies were again isolated from the males under $\mathrm{CO} 2$ anaesthesia and were left to recover for 2 hours before starting the experiments. For the experiments, approximately 20 females were transferred without anaesthesia into a BugDorm insect rearing cage $(24.5 \times 24.5 \times 24.5$ $\mathrm{cm})$ (MegaView Science Co., Ltd., TAIWAN) made of polyester netting. Two ø 50mm Petri dish plates containing Iberian fly food were placed in opposing corners of the cage and a small plastic cup cut from the cap of a $1.5 \mathrm{ml}$ Eppendorf tube containing the experimental odour, or the solvent control, was placed at the center of each food plate. For the geosmin experiments $5 \mu \mathrm{l}$ of geosmin (1:1000 dilution in mineral oil) was used as a stimulus. For the experiments done with yeast odour, $100 \mu \mathrm{l}$ of $400 \mathrm{mg} / \mathrm{ml}$ of baker's yeast in Milli-Q H2O was used. A nylon mesh was used to physically separate the flies from the odorant. All experiments started at 12:00 $\mathrm{h}$ Zeitgeber time $(+/-1 \mathrm{~h})$ and lasted for 16 hours $(+/-1 \mathrm{~h})$. Eggs were counted under a stereo microscope. An oviposition Preference Index (PI), was calculated by using the formula

$P I=($ Eggs on odour plate - Eggs on control plate $) /$ Total eggs 
PI could thus get values from -1 to +1 , signifying total avoidance and total preference of the geosmin plate, respectively.

We also developed an alternative version of the egg-laying assay (adapted from (31) and used in Figure S3D, E and F, Figure $3 \mathrm{~K}$, and Figure 4E). For this we used a single ø 50mm Petri dish plate containing Iberian fly food. The plate was split in two by a divider, the food was then melted by briefly heating the plates, and the experimental odour $(50 \mu \mathrm{l}$ of $1: 1000$ geosmin in Milli-Q H2O) and solvent control were mixed directly into the food. After the food had solidified again, the divider was removed. Five mated females were aspirated onto the plates and the Petri dish plate was placed back on top of the plates. Experimental duration and other parameters were as above. The main benefit of this version of the assay was that the PI variance was lower, which allowed us to use i) lower sample sizes and, ii) fewer flies per replicate, thus leading to a significantly improved experimental throughput. We ascertained that the behavioral phenotype was still solely due to olfaction (Figure S3D), and replicated the main results we obtained with the other assay (Figure S3E). PI was calculated as

$P I=($ Eggs on odour side - Eggs on control side $) /$ Total eggs

For the 1-methylindole experiments, we tried both 1:1000 and 1:10.000 concentrations. As a two-way ANOVA with genotype and concentration as factors showed a significant main effect for genotype, but not concentration, and there was no observable genotype $\mathrm{x}$ concentration interaction, we pooled both concentration groups for Figure $3 \mathrm{~K}$, with $\sim$ half of the flies for each genotype coming from each stimulus concentration.

\section{Egg-laying no-choice assay}

Fly collection, rearing and mating was performed similarly to the two-choice assay. For the no-choice assay, 5 females were aspirated without anaesthesia onto $\emptyset 50 \mathrm{~mm}$ Petri dish plates containing fly food, and the lid was placed on the plate. In the experiments where the effect of odorants on egg-laying quantity was tested, the stimuli were pipetted onto a small plastic cup cut from the cap of a $1.5 \mathrm{ml}$ Eppendorf tube. $50 \mu \mathrm{l}$ of geosmin was used as a stimulus. A nylon mesh was used for physically separating the flies from the odorant. All experiments started at 12:00 $\mathrm{h}$ Zeitgeber time $(+/-1 \mathrm{~h})$ and lasted for 16 hours $(+/-1 \mathrm{~h})$. Eggs were counted under a stereo microscope.

\section{Optogenetic four-field assay}

The four-field optogenetic assay was carried out essentially as described in (37). Crosses were made on normal fly food containing 1:500 all-trans retinal (Sigma-Aldrich, MO, USA), and eclosed females of the right genotype were collected into same-sex vials under cold anaesthesia, and reared in the dark on 1:250 all-trans retinal food, at $22 \mathrm{C}$ and $50 \%$ humidity. Approximately 20 female flies, aged 3-7 days were used for each experiment. The females were not specifically mated for the experiments, but they were producing fertilised eggs by the time of the experiments. The assay was performed on a circular arena of $10 \mathrm{~cm}$ diameter, and $3 \mathrm{~mm}$ height. Flies were transferred onto the arena without anaesthesia by using a vacuum pump. All experiments took place in darkness, at $25 \mathrm{C}$ and $50 \%$ humidity. To prevent the infrared backlight from affecting the temperature in the arena, the arena was mounted on a heat sink, and an airflow of $150 \mathrm{ml} / \mathrm{min}$ from the four corners of the arena to the centre was maintained.

A $617 \mathrm{~nm}$ wavelength LED (Red-Orange LUXEON Rebel LED; Luxeon Star LEDs, Brantford, Ontario, Canada) was used for the optical stimulation. The behaviour of the flies was recorded by using a camera (ROHS 1.3 MP B\&W Flea3, US 3.0 Camera; Point Grey, Richmond, BC, Canada) equipped with a long-pass $(800 \mathrm{~nm})$ filter (B\&W filter; Schneider Optics) set to capture at $30 \mathrm{~Hz}$, and controlled via a custom script written in Matlab. Only water was used for cleaning the arena.

\section{FlyPEZ assay}

The FlyPEZ assay was carried out as described in (41).

\section{Neuronal reconstructions in EM}

Two EM datasets were used: a full female adult Drosophila brain (FAFB) (11), and another partial adult female brain (hemibrain) (12). In FAFB, neuron skeletons were manually traced using CATMAID $(56,57)$, following the procedure as described in (11). The identification of antennal lobe glomeruli and their cognate PNs follows (14). PNs were traced to completion in the $\mathrm{LH}$, and all their presynapses and postsynapses were annotated. In the hemibrain, initial neuronal morphologies were generated by machine learning methods and then proofread by human experts. This process resolved any mistakes and merged additional processes, although neurons were not finished to completion. The average neuron completion rate in the $\mathrm{LH}$, as measured by the percentage of postsynaptic densities that belong to morphologies of a significant size, is only $19 \%$. The identification of synapses was an entirely automatic process (12) that differs from the synapse annotation process in CATMAID. For the reasons above, comparing connectivity between FAFB the hemibrain as to be done with care. The identification of PNs and LHAVlal neurons is as described in Scheffer $e t$ al. (12). The neuron morphologies and connectivity used are as released in neuPrintExplorer version 1.1

\section{DA2 PN downstream sampling in FAFB}

DA2 PN synapses were identified, and all postsynaptic nodes were annotated (consistent with the criteria described in (11)). Once completed, the full set of postsynaptic nodes for a single representative DA2 PN was randomised. Each postsynaptic node was then used as a starting point for tracing out a downstream partner. This sampling procedure was continued until all postsynaptic nodes were either connected to identifiable neurons, or excluded from further analysis due to not being able to connect it to a neuronal backbone (defined by the presence of visible microtubules) as a result of ambiguous features or missing EM sections. Overall, $74.11 \%$ of postsynaptic nodes were connected to an identifiable neuron. 913 914 915 916 917 918 919 920 921 922 .

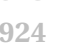

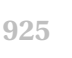
934 943 944 945 946 947 948 949
950 950
951

\section{5} 956 957 958
959 960 960 962 963 964 965 
Partner neurons were initially traced just enough to identify a soma, thus confirming whether the starting node belonged to a new or previously traced neuron. A sample of 15 LHNs of particular interest (the set in Figure 2) were then traced 'to completion'; all identifiable branches of the neuron were fully traced, and all incoming and outgoing synapses annotated (note: the neuropeptidergic brain-spanning neuron AVLP594 was only fully traced in the LH). With tracing completed, we were able to examine these neurons' complete morphology (manually identifying the primary neurite, dendrites, and axon for each), as well as their PN inputs within the LH.

This basic sampling procedure was repeated for the axons of selected third order neurons (LHAV1a1\#1 and PV6a3\#1). However, the process was not continued to completion for these neurons (Figure S4A and D).

\section{Potential DA2 axo-axonic connectivity in FAFB}

To assess whether or not the DA2-DL4 connectivity was specific, we checked which PN skeletons pass within $1 \mu \mathrm{m}$ of a DA2 output synapse in the LH (in the right LH, approximately $86 \%$ of PN-PN axo-axonic synapses occur within this threshold-data not shown). Each instance where a PN skeleton was within this $1 \mu \mathrm{m}$ radius was counted as a single potential synapse. This potential connectivity was then compared to the observed DA2 PNs axo-axonic connectivity.

\section{Clustering of FAFB neuron morphologies}

Neurons downstream of the completed DA2 PN were first divided into four broad groups: LHONs, LHLNs, PNs, and others. PNs were excluded from further analysis, and each broad group of neurons analysed separately. The nat.nblast package (https://github.com/natverse/nat.nblast) was used for both NBLASTing $(16,58,59)$ and hierarchical clustering of the neurons. More specifically, within the broad groups, each neuron was split into a primary neurite (approximated by taking the longest unbranching segment of the neuron), and the rest (the complement of the primary neurite approximation). Both parts of each neuron were then converted to dot properties representations $(16,58)$. The NBLAST algorithm $(16,58)$ was used to generate two all by all similarity matrices; one for the primary neurites, and one for the rest of the neurons. The obtained matrices were then combined by taking the weighted element-wise mean of both matrices, so that the primary neurite was assigned a weight of 0.8 and the rest of the neuron a weight of 0.2 . This was done to i) more closely match the manual annotation system of LHNs (that uses the primary neurite tract as the first distinguishing feature in a tri-level hierarchical scheme) (17), and ii) deal with the incompleteness of a large proportion of the neurons. The NBLAST similarity matrices were then converted to distance matrices, and hierarchical clustering was performed by using the average linkage method. Cut heights were determined separately for each broad group (LHON, LHLN, other) after manually assessing the cluster groups.

\section{Neuron and neuropil nomenclature}

Annotation of neuronal types was based on Bates et al. (14) 1020 and Scheffer et al. (12) (for LHNs) and Namiki et al. (51) (for 1021 DNs). For the cases in which there is more than one individ- 1022 ual per type, each individual has been given a unique name 1023 by adding '\#<number>' after the type name. Image data for 1024 light level type example skeletons from FlyCircuit for pre- 1025 viously described types (15) can be browsed by searching 1026 for the neuron identifier at http://www.virtualflybrain.org/. 1027 Neuropil nomenclature was based on (18) for the brain, and 1028 (60) for the VNC.

\section{In silico anatomy screen}

A confocal stack of an R85E04 brain was registered onto a 1031 JFRC2 template brain (5), and the axonal arbors of the DA2 1032 PNs were converted into a binary mask with the Segmenta- 1033 tion Editor tool in the Fiji software (NIH, Bethesda, USA). The 1034 mask was then used as an ROI to look for driver lines with 1035 expression overlapping with the DA2 axons in the LH. This 1036 was done by overlaying Janelia FlyLight GAL4 lines (5) with 1037 expression in the LH with the mask and visually assessing the 1038 overlap. Each line was scored for goodness of overlap, and the 1039 neurons in the best lines were identified (17), and then cross- 1040 identified in the LH Split-GAL4 lines (36), where possible.

\section{Confocal microscopy}

A Zeiss 710 confocal microscope was used for image acqui- 1043 sition. Brains were imaged at 768 x 768, or 2048x1024 (AL 1044 closeup), pixel resolution in $1 \mu \mathrm{m}$ slices (voxel size: $(0.46 \mathrm{x} 1045$ $0.46 \times 1 \mu \mathrm{m})$ using an EC Plan-Neofluar 40x/1.30 oil immer- 1046 sion objective (Carl Zeiss AG, Jena, Germany) and 0.6 zoom 1047 factor. All images were acquired at 16 bit colour depth. $\quad 1048$

\section{Image registration}

Image registration for the confocal data was carried out 1050 according to (61). In brief: the presynaptic marker Bruch-1051 pilot (labeled by nc82) was used as a basis for performing 1052 an intensity-based non-rigid warping registration (62) 1053 onto a template brain (JFRC2 or JFRC2013, available here: 1054 https://github.com/jefferislab/BridgingRegistrations). 1055 The registration procedure itself was performed by using 1056 the cross platform Computational Morphometry Toolkit 1057 software (http://www.nitrc.org/projects/cmtk). Bridging 1058 registrations were used for transforming neurons from one 1059 template brain to another $(11,59)$ by using the nat.flybrains 1060 (https://github.com/natverse/nat.flybrains) and elmr 1061 (https://github.com/natverse/elmr) R packages. A similar 1062 template was derived from the nc82 expression pattern 1063 in the VNC of an example female Canton S fly imaged by 1064 the FlyLight Project team (template available here: https: 1065 //github.com/VirtualFlyBrain/DrosAdultVNSdomains/ 1066 blob/master/template/Neuropil_185.nrrd). Our VNC align- 1067 ment pipeline was adapted from (60). Briefly: confocal 1068 VNC stacks were first converted to an 8-bit nrrd file format, 1069 preprocessed using the nc82 reference channel to normalize 1070 contrast across samples, rotated to approximately orient the 1071 VNC along the anterior-posterior axis, and then the channels 1072 
1073 were aligned to the template by nonrigid warping (62) using 1074. the Computational Morphometry Toolkit.

\section{Image processing for DN lines}

1076 Neuron tracing was carried out semi-manually using Amira 10775.4 .3 (Visage Imaging, Fuerth, Germany). Volume rendering 1078 was performed using Amira 'generate surface' function. We 1079 first detected the signal with the Amira 'Interactive Thresh1080 olding' function. We then corrected any false detection by 1081 manual tracing. Using this image as a mask, we obtained the 1082 final masked images shown in the figures using a custom1083 made program written in MATLAB and the image process1084 ing toolbox (MathWorks, Natick, MA, USA). The contrast and 1085 brightness of images were modified in Image J (National In1086 stitutes of Health, Bethesda, MD). Confocal image stacks of 1087 split-GAL4 expression patterns in the brain were aligned to 1088 standardized brain template JFRC2013 (see above).

\section{Light versus EM comparisons of neuron mor- 1090 phology}

\section{Immunohistochemistry}

Immunohistochemistry with antibodies was done similarly to (10), and the chemical labeling similarly to (6), with the exception of an overnight blocking step being used for antibody stainings. Primary antibodies used were: 1:20 mouse antinc82 (DSHB, University of Iowa, USA), 1:1600 chicken antiGFP (ab13970, Abcam, Cambridge, UK), 1:200 rabbit antiGABA (A2052, Sigma-Aldrich, MO, USA), and 1:400 mouse anti-ChAT (4B1, DSHB, University of Iowa, USA). Secondary antibodies were: Alexa-488 Goat anti-chicken, Alexa-568 Goat anti-Rabbit, Goat anti-mouse 633, all 1:800 (Life Technologies, Carlsbad, CA). For the chemical labeling, 1:1000 concentrations of SNAP-Surface 488 (NEB \#S9124, New England Biolabs, Ipswich, MA), TMR Halo (G8252, Promega, Madison, WI) were used. Finally, brains were mounted on charged slides (Menzel-Glaeser, Braunschweig, Germany) using Vectashield (Vector Laboratories) as the mounting medium.

\section{Electrophysiology}

In vivo patch-clamp recordings from the DA2 projection neurons were carried out as described in (17) using the R85E04 driver line and mCD8::GFP to label the neurons. Analysis 1126 of recordings used the open source gphys R (CRAN, http: 1127 //www.r-project.org) package (see http://jefferis.github.io/ 1128 gphys).

\section{In vivo calcium imaging}

Functional imaging experiments on LHAVlal neurons were 1131 performed on flies containing two copies of UAS-GCaMP3 1132 (at attP18 and attP40) driven by LH728 Split-GAL4 driver. 1133 GCaMP3 was used instead of newer versions of GCaMP for its 1134 higher baseline fluorescence which allowed easier identifica- 1135 tion of the neurons. Flies were placed into custom built hold- 1136 ers, leaving the head and thorax exposed, under CO2 anaes- 1137 thesia and secured in place with UV curable glue (Kemxert, 1138 KOA 300). Wax was used for securing the legs and the pro- 1139 boscis. A window was then cut into the head capsule with 1140 sharp forceps, and trachea and air sacks were removed in 1141 order to uncover the brain. Fly brains were bathed in exter- 1142 nal saline ([94]) adjusted to $275 \mathrm{mM}$ and $7.3 \mathrm{pH}$, and bub- 1143 bled with 5\% CO2. The saline had the following composi- 1144 tion (Concentration, $\mathrm{mM}$ ): $\mathrm{NaCl} 104.75 ; \mathrm{KCl} 5$; NaH2PO4 1; 1145 $\mathrm{MgCl} 2.6 \mathrm{H} 2 \mathrm{O}$ 1; $\mathrm{CaCl} 2.2 \mathrm{H} 2 \mathrm{O}$ 1; NaHCO3 26; TES 5; glucose 1146 10; trehalose 10. The antennae were left under the holder 1147 so that they could be exposed to odour stimuli. A custom- 1148 built setup based on the Sutter (Novato, CA) Movable Ob- 1149 jective Microscope with a Zeiss W Plan-Apochromat 20x/1.0 1150 objective was used for the two photon imaging. A Coherent 1151 (Santa Clara, CA) Chameleon Vision Ti-Sapphire provided ex- 1152 citation, and image acquisition was controlled by ScanIm- 1153 age software (63). Image acquisition and odour delivery were 1154 triggered by a separate PC via Igor Pro software (Wavemet- 1155 rics, Lake Oswego, OR) running Neuromatic. Images were 1156 captured at $8 \mathrm{~Hz}$ at $265 \times 255$ pixel, and two photon excitation 1157 was provided at $900 \mathrm{~nm}$. Odour stimulation was performed 1158 largely similarly to (24). Odour delivery started at $3000 \mathrm{~ms} 1159$ after the beginning of a trial and lasted for $2000 \mathrm{~ms}$. Im- 1160 age analysis was performed with custom scripts written in 1161 R employing the open source scanimage package (see https: 1162 //github.com/jefferis/scanimage, 10.5281/zenodo.1401028). 1163 Data was both manually checked for motion artifacts, and ex- 1164 cluded from the analysis if there were larger than $5 \% \mathrm{dF} / \mathrm{F} 1165$ peaks during the baseline recording epoch, or if there were 1166 not larger than $5 \% \mathrm{dF} / \mathrm{F}$ responses to any of the tested odours 1167 during the stimulation epoch.

\section{Statistical analysis}

All statistical analysis was performed in R (https://cran. 1170 r-project.org/). Shapiro-Wilk or Kolmogorov-Smirnov tests 1171 were used for assessing normality of distributions. Normally 1172 distributed data was then analysed by using One or Two- 1173 way ANOVAs, Welch's one or two-sample t-tests, or Paired 1174 samples t-tests, whereas non-normally distributed data with 1175 Kruskal- Wallis rank sum tests, Wilcoxon rank sum tests, 1176 and Wilcoxon signed rank tests, where appropriate (see also 1177 Supplementary Table 1). For the egg-laying two-choice be- 1178 havioural experiments (with R85E04 and LH728), power test- 1179 ing was done with effect size estimates based on data ob- 1180 tained on preliminary data on wild type and anosmic mu-1181 tants (Ir8a1; Ir25a2; Orco1, Gr63a1) (values used for power 1182 
test estimates were wild type mean $\mathrm{PI}=-.45, \mathrm{SD}=.6$; anosmic mean $\mathrm{PI}=0, \mathrm{SD}=.6$, which gives an effect size estimate of Cohen's $d=0.75$ ), and based on this the required sample size for the experiments was 38.66, taking into account the Bonferroni corrected significance values. The power size estimations were done with the R package pwr (https://cran. r-project.org/web/packages/pwr).

The randomization tests for comparing DA2 downstream target morphology (see also 'Clustering of neuron morphology' below) to the number of DA2 inputs (Figure S2F) was conducted with custom R scripts. First, the coefficient of variation (CV) of DA2 inputs for each morphological cluster was calculated as

$$
\begin{array}{r}
C V=\mathrm{SD} \text { of DA2 inputs for cluster } / \\
\text { Mean of DA2 inputs for cluster }
\end{array}
$$

The DA2 input numbers were then randomly reassigned to clusters repeatedly $(\mathrm{n}=10.000)$, and new CVs were calculated for each iteration. Finally, the weighted mean (weighted by the proportion of neurons belonging to the cluster) of the original CVs were compared to the distribution of mean $\mathrm{CV}$ values obtained by the reshuffling. This was not done on 'other' neurons as only a few morphological clusters had multiple neurons in them, which makes it impossible to assess the variability of a cluster.

The randomization tests for aversion index (AI) values of completed LHNs were conducted with custom R scripts. First, the connectivity matrix of all excitatory uPNs (with known valence) was obtained via the $\mathrm{R}$ package catmaid (https://github.com/natverse/rcatmaid). In the cases of multiple sister PNs innervating the same glomerulus, the PNs were collapsed together into a PN type by taking the sum of their connections. All PN types were then assigned a valence ("aversive" or "not aversive") based on earlier literature. After removing PNs of unknown valence, AI values for the LHNs were calculated as the ratio of the sum of connections from aversive PNs and the total sum of connections from excitatory uPNs. For the randomisation tests, DA2 PNs were excluded from the sample to avoid bias, and new (nonDA2) AI values were calculated. The valence labels were then randomly reshuffled $(n=1000)$ and the AI values recalculated. The number of aversive and not aversive PN types was held constant, and the same as for the original data throughout. For each LHN, the observed distribution of random AI values was then compared to the original value, and the neuron was considered to significantly integrate aversive input if the observed AI value was higher than $95 \%$ of the values obtained by reshuffling.

Behavioural and imaging data throughout the paper are presented as notched box plots. The box represents the interquartile range of the sample (IQR, 25th - 75th percentiles) and is split by the median line. The whiskers extend to $1.5 \mathrm{x}$ IQR beyond the box and the notches represent the 95\% confidence interval for the sample median. The points mark individual sample points and asymmetrical notches indicate skewed distributions.

\section{Data availability}

The FAFB reconstructed neurons will be shared with the Virtual Fly Brain project upon publication and will be available from (https://fafb.catmaid.virtualflybrain.org/) . In addition, 1239 we are providing the skeletons as SWC files and a connectiv- 1240 ity matrix as supplementary files. 